\title{
Synthesis of anomeric sulfonamides and their behaviour under radical-mediated bromination conditions
}

\author{
Katalin Czifrák, László Somsák* \\ Department of Organic Chemistry, University of Debrecen, PO Box 20, H-4010 Debrecen, Hungary
}

\section{A R T I C L E I N F O}

10 Article history:

Received 10 October 2008

Received in revised form 5 November 2008 Accepted 5 November 2008

Available online $\mathrm{xxxx}$

\section{Keywords:}

Sulfonamide

Glycopyranosyl

Bromination

Radical

\begin{abstract}
A B S T R A C T
Q-Peracetylated methyl 3्र-(D-glycopyranosylthio)propanoates of $\beta$-D-gluco, and $\alpha$ - and $\beta$-D-galacto configurations were oxidized to the corresponding S,S-dioxides (sulfones) by Oxone ${ }^{\circledR}$ or MCPBA. Oxidation of the $\beta$-D-gluco derivative with $\mathrm{H}_{2} \mathrm{O}_{2} / \mathrm{Na}_{2} \mathrm{WO}_{4}$ gave the corresponding S-oxide (sulfoxide). DBU-induced elimination of methyl acrylate from the $\beta$-D-gluco and $\beta$-D-galacto configured $S_{K} S$-dioxides (sulfones) gave O-peracetylated $\beta$-D-glycopyranosyl-1-C-sulfinates which, on treatment with $\mathrm{H}_{2} \mathrm{NOSO}_{3} \mathrm{H}$, furnished the corresponding $\beta$-D-glycopyranosyl-1-C-sulfonamides. Radical-mediated bromination of the protected methyl 3-( $\beta$-D-glycopyranosylthio)propanoate S,S-dioxides gave mixtures of $1-C$ - and 5-C-bromoglycosyl compounds. Similar brominations of the Q-peracetylated $\beta$-D-glycopyranosyl-1-C-sulfonamides resulted in the formation of $\alpha$-D-glycopyranosyl bromides and 1- $C$ - and 5-C-bromoglycosyl sulfonamides. A rationale for these observations was proposed. Methyl 3-( $\beta$-D-glucopyranosylthio)propanoate, its S,S-dioxide, and $\beta$-D-glucopyranosyl-1-C-sulfonamide proved inefficient when tested as inhibitors of rabbit muscle glycogen phosphorylase $b$.
\end{abstract}

() 2008 Elsevier Ltd. All rights reserved.

\section{Introduction}

As part of an ongoing project to design and synthesize glycoenzyme inhibitors, we have prepared several compounds ${ }^{1-4}$ with glycogen phosphorylase (GP) inhibitory activity ${ }^{5,6}$ from (3,4,5,7-tetra$O$-acyl- $\alpha$-D-glyco-hept-2-ulopyranosyl bromide)onamides (A, Chart $1 \hat{)}$. Among them, compound $\mathbf{C}$ was shown to have moderate inhibition against rabbit muscle GPb (RMGPb). ${ }^{4}$ Since GP is a validated target for the treatment of type 2 diabetes mellitus many efforts<smiles>NC(=O)C1(Br)OCCC(OC(F)(F)F)C1OP(=O)(O)OC(F)(F)F</smiles>

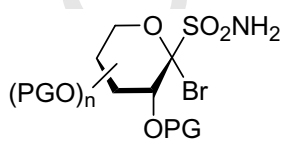

$P G=A c, B z$

A

B

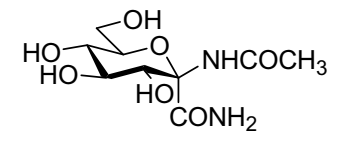

C

$$
\text { measured }^{4} K_{\mathrm{i}}=310 \mu \mathrm{M} \quad \text { predicted }^{9} K_{\mathrm{i}}=0.59 \mu \mathrm{M}
$$

Chart 1. are devoted to finding new inhibitors (GPIs). ${ }^{7,8}$ To this end, among others, computational chemistry is in the forefront of GPI design. ${ }^{8}$ By using 4D-QSAR methods, it was predicted that compound $\mathbf{D}$, a sulfonyl analogue of $\mathbf{C}$, might be a very efficient GPI with an inhibition constant in the nanomolar range. ${ }^{9}$ Therefore, we envisaged to prepare $\mathbf{D}$ from $\mathbf{B}$ in a way analogous to the chemistry ${ }^{2}$ applied to obtain $\mathbf{A}$ and its further transformations to $\mathbf{C}$.

To the best of our knowledge, the necessary starting materials to get $\mathbf{A}$, that is, $\mathrm{N}$-unsubstituted anomeric sulfonamides, have not yet been described. The only analogous compound is the $N, N$-diethylsulfonamide in the $\alpha$-anomeric position of $N$-acetyl-Dglucosamine obtained by substituting $\mathrm{Et}_{2} \mathrm{NH}$ in the corresponding glycosylsulfenyl chloride or bromide and subsequent oxidation. ${ }^{10}$

In this paper we disclose our experiences in the preparation of glycopyranosyl sulfonamides and their reactivity under radicalmediated bromination conditions.

\section{Results and discussion}

A generally used method for the preparation of sulfonamides is the ammonolysis of the corresponding sulfonylchlorides. At the commencement of our work, glycosyl sulfonyl chlorides (e.g., 9) were not known, therefore chlorination of sugar sulfonate salt $\mathbf{4}$ was envisaged. Several sulfonic acids and their salts attached to a sugar skeleton were described. ${ }^{11-13, \dagger}$ The general method for

\footnotetext{
${ }^{\dagger}$ For a more detailed list of citations see Ref. 23 in a paper by Knapp et al. ${ }^{10}$
}

\footnotetext{
Corresponding author. Tel.: +36 52512 900/22348; fax: +36 52453836 .

E-mail address: somsak@tigris.unideb.hu (L. Somsák).
} 


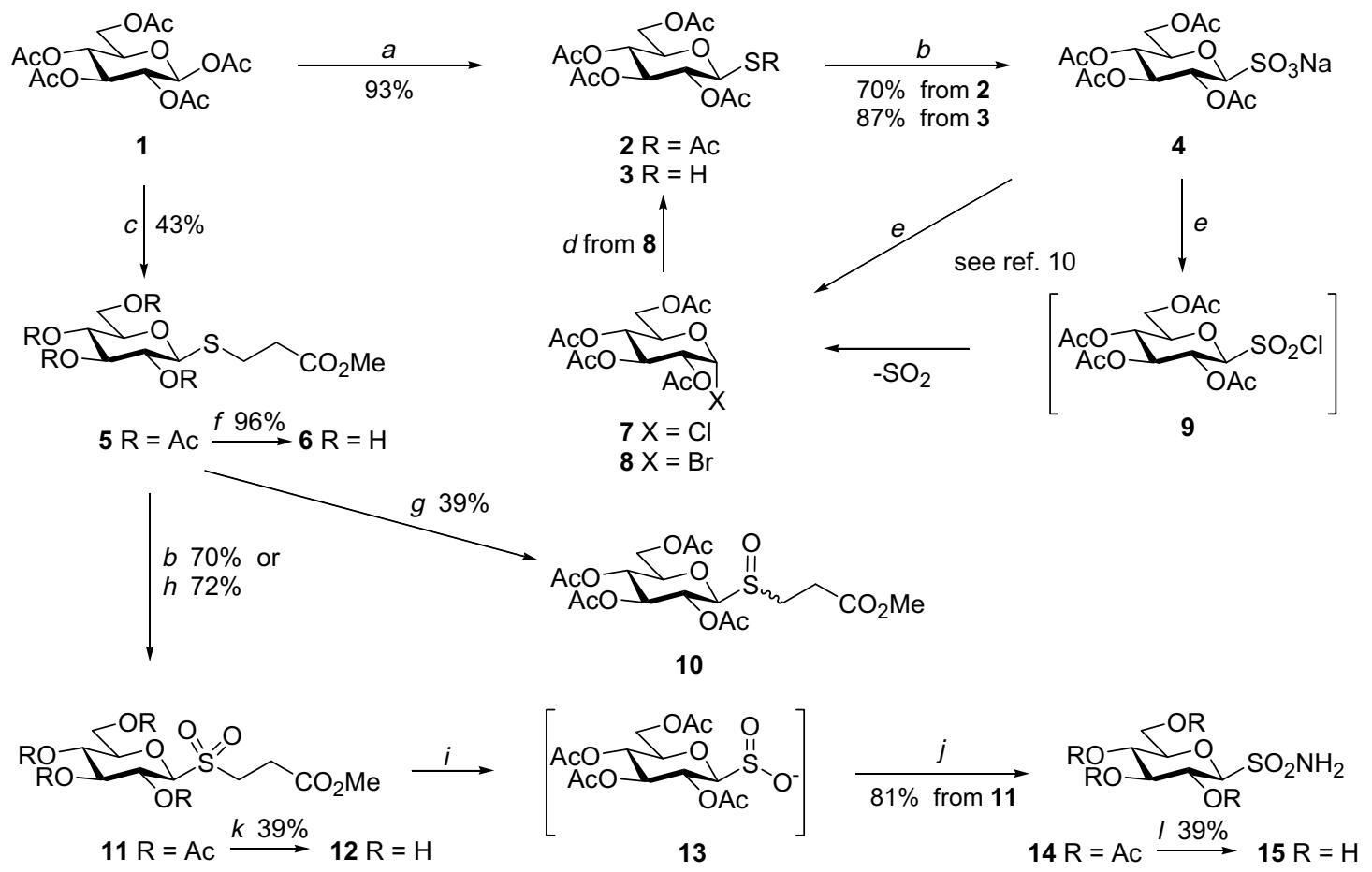

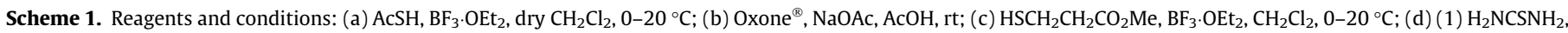

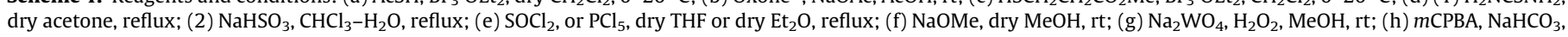
$\mathrm{CH}_{2} \mathrm{Cl}_{2}, 0-2{ }^{\circ} \mathrm{C}$; (i) $\mathrm{DBU}, \mathrm{CHCl}_{3}, \mathrm{rt}$; (j) $\mathrm{H}_{2} \mathrm{NOSO}_{3} \mathrm{H}$, water, rt; (k) AcCl, dry $\mathrm{MeOH} \mathrm{rt}$; (l) $\mathrm{NH}_{4} \mathrm{OH}, \mathrm{MeOH}$, rt.

obtaining such compounds was a nucleophilic displacement by AcSK in the corresponding sugar derivative followed by oxidation, ${ }^{13}$ and it was also extended to the anomeric position. ${ }^{14}$ Thus, to get sodium sulfonate 4 (Scheme 1 ), either O-peracetylated $1-S$-acetyl-1-thio- $\beta$ D-glucopyranose ${ }^{15}$ (2) prepared from 1,2,3,4,6-penta- $O$-acetyl- $\beta$-Dglucopyranose (1) and thiolacetic acid ${ }^{16}$ or O-peracetylated 1-thio$\beta$-D-glucopyranose (3) obtained by the conventional literature method $^{17,18}$ from 8 was oxidized by Oxone ${ }^{\circledR}{ }^{14}$ The corresponding triethylammonium salt ${ }^{10}$ was made from 2 by DMDO oxidation followed by chromatography in the presence of $\mathrm{Et}_{3} \mathrm{~N}$. Reactions of 4 under usual chlorination conditions (neat $\mathrm{SOCl}_{2}$, reflux; THF, $\mathrm{SOCl}_{2}$; THF$\mathrm{Et}_{2} \mathrm{O}, \mathrm{PCl}_{5}$ ) gave several compounds which were not separated. 80 Experiments to add nucleophiles to the crude chlorination mixtures with the hope of trapping any sulfonyl chloride formed were unsuccessful. Knapp et al. isolated the corresponding glycosyl chloride 7 (70\%) from the chlorination mixture of their triethylammonium sulfonate, ${ }^{10}$ and accounted for the formation of 7 by $\mathrm{SO}_{2}$ extrusion from the unstable intermediate sulfonyl chloride $\mathbf{9}$. For these reasons other possibilities were sought for the preparation of the target sulfonamides.

Various sulfonamides were prepared by reactions of sulfinate salts with electrophilic nitrogen sources such as hydroxylamine$O$-sulfonic acid ${ }^{19,20}$ or bis(2,2,2-trichloroethyl)azodicarboxylate. ${ }^{21}$ The preparation of the required anomeric sulfinate 13 was effected by base-induced $\beta$-elimination from sulfon $\mathbf{1 1}$ which was obtained by oxidation of thioglucoside $\mathbf{5}^{22,23}$ by either Oxone ${ }^{\circledR}$ or mCPBA. Application of $\mathrm{H}_{2} \mathrm{O}_{2} / \mathrm{Na}_{2} \mathrm{WO}_{4}{ }^{20,21}$ to oxidize $\mathbf{5}$ gave sulfoxides $\mathbf{1 0}$ (two TLC spots of very similar mobility) from which one isomer could be isolated in pure state in moderate yield. As an alternative route to sulfinates, the reductive cleavage of benzothiazolyl sulfones was proposed; ${ }^{21}$ however, practically no change could be observed in the reaction of O-peracetylated $\beta$-D-glucopyranosyl benzothiazol-2-yl sulfone ${ }^{24}$ with $\mathrm{NaBH}_{4}$ in THF/MeOH for $\sim$ one week even at elevated temperature. Sulfinate 13, without being purified, was treated with $\mathrm{H}_{2} \mathrm{NOSO}_{3} \mathrm{H}$ in water to give the desired sulfonamide $\mathbf{1 4}$ after chromatographic purification in $81 \%$ yield for the two steps.

For the preparation of the D-galacto configurated derivative 23 (Scheme 2), the corresponding thioglycoside had to be prepared first. $\mathrm{BF}_{3}$ catalyzed reaction of 1,2,3,4,6-penta- $O$-acetyl- $\beta$-D-galactopyranose (16) with methyl 3-sulfanylpropanoate gave the $\alpha$-thiogalactoside $\mathbf{1 8}$ in moderate yield. To get the $\beta$-anomer, 2,3,4,6-tetra- $O$-acetyl-1-thio- $\beta$-D-galactopyranose ${ }^{25,26}$ (17) was

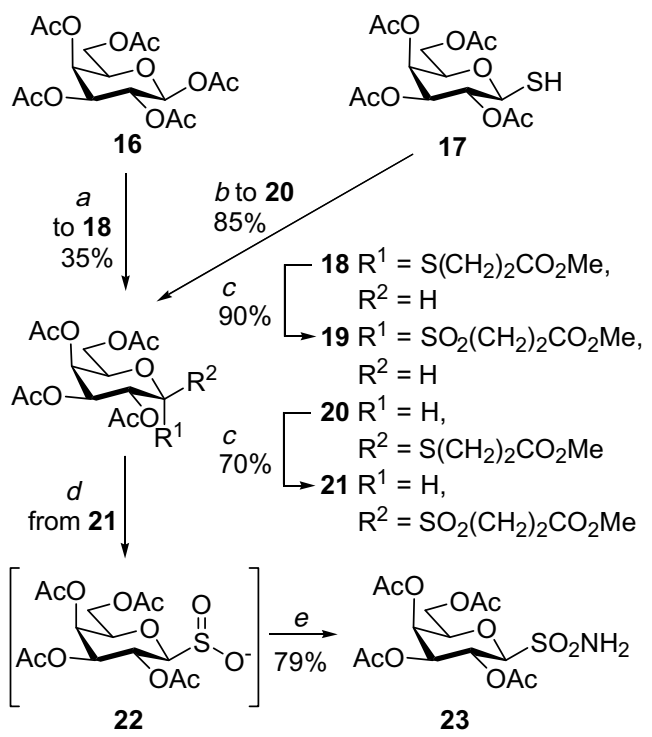

Scheme 2. Reagents and conditions: (a) $\mathrm{HSCH}_{2} \mathrm{CH}_{2} \mathrm{CO}_{2} \mathrm{Me}, \mathrm{BF}_{3} \cdot \mathrm{OEt}_{2}, \mathrm{CH}_{2} \mathrm{Cl}_{2}$ 0-20 ${ }^{\circ} \mathrm{C}$; (b) $\mathrm{BrCH}_{2} \mathrm{CH}_{2} \mathrm{CO}_{2} \mathrm{Me}, \mathrm{Et}_{3} \mathrm{~N}, \mathrm{CH}_{2} \mathrm{Cl}_{2}, \mathrm{rt}$; (c) Oxone ${ }^{\circledR}, \mathrm{NaOAc}, \mathrm{AcOH}, \mathrm{rt}$; (d) $\mathrm{DBU}, \mathrm{CHCl}_{3}$, rt; (e) $\mathrm{H}_{2} \mathrm{NSO}_{3} \mathrm{H}$, water, rt. 
Table 1

Radical-mediated bromination of sulfonyl derivatives

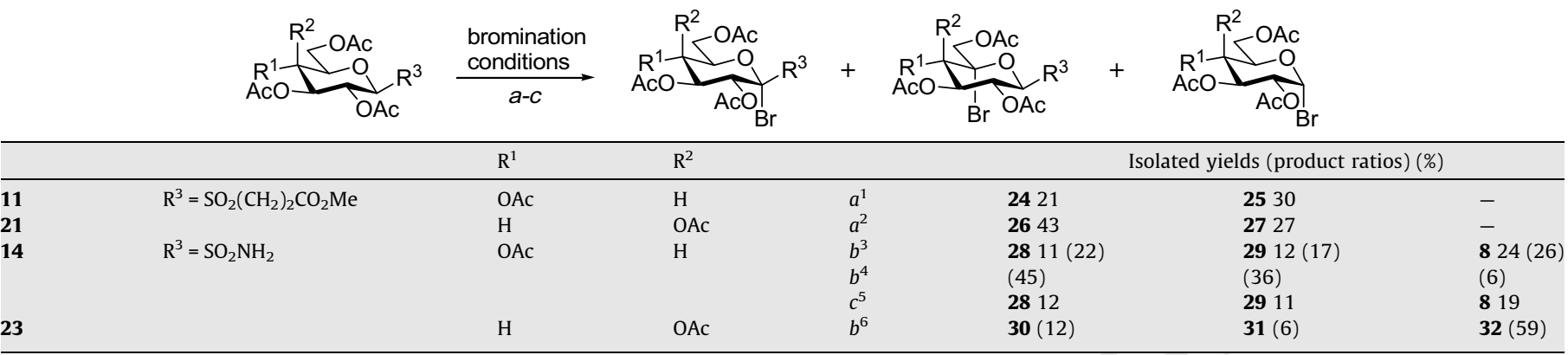

Bromination conditions:

(a) $\mathrm{Br}_{2}, \mathrm{CCl}_{4}, \mathrm{~K}_{2} \mathrm{CO}_{3}, h v$, reflux. ${ }^{27}$

(b) $\mathrm{Br}_{2}, \mathrm{CHCl}_{3}, \mathrm{~K}_{2} \mathrm{CO}_{3}, h v$, reflux. ${ }^{2}$

(c) $\mathrm{Br}_{2}, \mathrm{PhCF}_{3}, \mathrm{~K}_{2} \mathrm{CO}_{3}, h v$, reflux. ${ }^{30}$

${ }^{1}$ Reaction time $3 \mathrm{~h}$.

${ }^{2}$ Reaction time $2 \mathrm{~h}$.

${ }^{3}$ Conversion $65 \%$ after $8 \mathrm{~h}$ with $0.6 \mathrm{~g}$ starting material.

${ }^{4}$ Conversion $87 \%$ after $4 \mathrm{~h}$ with $0.1 \mathrm{~g}$ starting material. Product ratio calculated from the ${ }^{1} \mathrm{H}$ NMR spectrum of the crude mixture.

${ }^{5}$ Conversion $81 \%$ after $4 \mathrm{~h}$.

${ }^{6}$ Conversion $77 \%$ after $4 \mathrm{~h}$. Product ratio calculated from the ${ }^{1} \mathrm{H}$ NMR spectrum of the crude mixture.

reacted with methyl 3-bromopropanoate in the presence of $\mathrm{Et}_{3} \mathrm{~N}$, and the required compound $\mathbf{2 0}$ was obtained in good yield. The anomeric configuration of these derivatives was clearly indicated by the vicinal coupling constants: ${ }^{3} J_{1,2}=5.3 \mathrm{~Hz}$ for 18 and ${ }^{3} J_{1,2}=9.2 \mathrm{~Hz}$ for 20 . Oxidation of thiogalactosides 18 and 20 by Oxone $^{\circledR}$ afforded the corresponding sulfones 19 and 21, respectively, in good yields $\left({ }^{3} J_{1,2}=2.6 \mathrm{~Hz}\right.$ for 19 and ${ }^{3} J_{1,2}=9.2 \mathrm{~Hz}$ for 21). Treatment of the $\beta$-anomeric sulfone 21 with $\mathrm{DBU}$ followed by reaction of the non-isolated sulfinate 22 with $\mathrm{H}_{2} \mathrm{NOSO}_{3} \mathrm{H}$ gave the expected sulfonamide $\mathbf{2 3}$ in 79\% overall yield for the two steps. Similar base-induced reaction of the $\alpha$-anomer 19 resulted in a mixture from which no discrete product could be isolated.

Radical-mediated bromination of various carbohydrate derivatives proved a useful tool to produce versatile compounds for further functionalization. ${ }^{27}$ Several pyranoid monosaccharide substrates showed excellent regio- and stereoselectivities under the bromination conditions to give either 1 - $C$-bromo- or 5 - $C$-bromo derivatives of pyranoid rings with the bromine substituents in axial positions. On the other hand, a study on bromination of phenyl 2,3,4,6-tetra-O-acetyl- $\beta$-D-glucopyranosyl sulfone demonstrated similar reactivities for the 1 - and 5-positions ( $\mathrm{C}-1$ bromide $48 \%$, C-5 bromide $38 \%)^{28}$

With these preliminaries in mind, it was to be expected that bromination (Table 1 ) of the gluco (11) and galacto (21) sulfones would give isomeric axial bromides 24 and $\mathbf{2 5}$, as well as $\mathbf{2 6}$ and 27, respectively. Bromination of sulfonamides 14 and 23 under different conditions gave varying proportions of the corresponding $\alpha$ D-glycopyranosyl bromides $\mathbf{8}$ and $\mathbf{3 2}$, respectively, the ratio âso being dependent on the scale of the reaction. The ring brominated derivatives $\mathbf{2 8}$ and $\mathbf{2 9}$, as well as $\mathbf{3 0}$ and $\mathbf{3 1}$, respectively, were formed as minor products when the reaction was performed on a larger scale. Compounds $\mathbf{2 8}$ and $\mathbf{2 9}$ were isolated by chromatography; however, the very similar chromatographic behaviour of $\mathbf{3 0}$ and 31 did not allow these derivatives to be prepared in pure state. The position of the bromine substitution in the sugar ring followed clearly from the coupling pattern of the ${ }^{1} \mathrm{H}$ NMR spectra of these compounds.

\footnotetext{
${ }^{\star}$ In the literature there are some examples with a similar outcome to the present one: the corresponding $\alpha$-D-glycosyl bromides were formed on radical mediated bromination of $\mathrm{O}$-peracetylated phenyl $\beta$-D-glucopyranosyl sulfoxide, ${ }^{28}$ cellobiosyl pyperidine $^{29}$ and $\beta$-D-glucopyranosyl isothiocyanate. ${ }^{29}$
}

These observations can be correlated with the relative radical stabilization (RRS) factors of substituents (Table 2) as in earlier explanations for the regioselectivity of radical-mediated bromination of sugar derivatives. ${ }^{27}$ As shown in Figure 1a, the radical stabilization is highest in position 1 of the sugar ring ( $R R S=8.3$; for positions 2-4 the OAc substituent is characterized by an RRS value of $0.9^{31}$ ). Although the radical in position next to the $\mathrm{CO}_{2} \mathrm{Me}$ group seems to be better stabilized than C-5 (RRS 7.9 vs 6.5), the observed higher reactivity of position 5 might be attributed to its tertiary character (as compared to the secondary carbon in the aglycon) which is not taken into consideration in the calculation of the RRS value. However, bromination in the methylene groups of the aglycon cannot be excluded, as very minor bromine-containing spots were observed by TLC in the reaction mixtures. Compounds with a carbon substituent at position one (for the present particular comparison a carboxamido group, but also other anhydro-aldonic acid derivatives) showed an exclusive selectivity for that position ${ }^{2,32,33}$ as it can be concluded from the RRS values ${ }^{27}$ (Fig. 1b). In the bromination of sulfonamides the relative reactivity of positions 1 and 5 was similar to those of the sulfones (Fig. 1a). However, the formation of glycosyl bromides needs further considerations. We propose that in the bromination of sulfonamides (Fig. 1c) the bromine radical may also abstract a hydrogen from the $\mathrm{SO}_{2} \mathrm{NH}_{2}$ moiety as illustrated in I. Further $\beta$-fragmentation of the intermediate sulfonamidyl radical II might give glycosyl radi$\mathrm{cal}^{34}$ III which, on bromine abstraction from the reagent, could give the glycosyl bromide IV with high stereoselectivity. The preponderance of this pathway may be attributed to the relatively free

Table 2

Selected relative radical stabilization factors ${ }^{31}$ of substituents $\left(R_{R} S_{X}\right)$ and their combined values $\left(\mathrm{RRS}_{\mathrm{O}, \mathrm{X}}\right)$ at positions 1 and 5 of the pyranoid ring

\begin{tabular}{lrc}
\hline $\mathrm{X}$ & $\mathrm{RRS}_{\mathrm{X}}$ & $\mathrm{RRS}_{\mathrm{O}, \mathrm{X}}$ \\
\hline $\mathrm{CH}_{3}\left(=\mathrm{CH}_{2} \mathrm{OAc}\right)$ & 2.3 & 6.5 \\
$\mathrm{OMe}(=\mathrm{O}$-ring) & 4.5 & \\
$\mathrm{SO}_{2} \mathrm{Ph}$ & 4.5 & 8.3 \\
$\mathrm{COOMe}$ & 7.9 & 11.3 \\
$\mathrm{CN}$ & 8.6 & 11.9 \\
$\mathrm{COMe}$ & 10.2 & 13.2
\end{tabular}

$\mathrm{X}$ represents the substituent in position 1 or 5 of the pyranoid ring while 0 stands for the ring oxygen (its numerical value equated with that of $\mathrm{OMe}$ ). The combined effects were calculated by using the expression: ${ }^{31} 0.03 \times \mathrm{RRS}_{\mathrm{O}, \mathrm{X}}=1-(1-0.03 \times$ $\left.\mathrm{RRS}_{\mathrm{OMe}}\right)\left(1-0.03 \times \mathrm{RRS}_{\mathrm{X}}\right)$. 
a

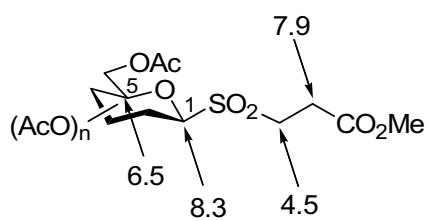

b

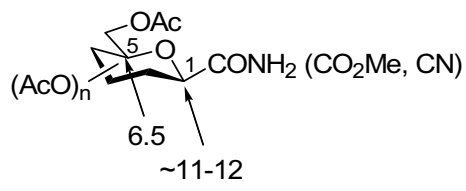

C

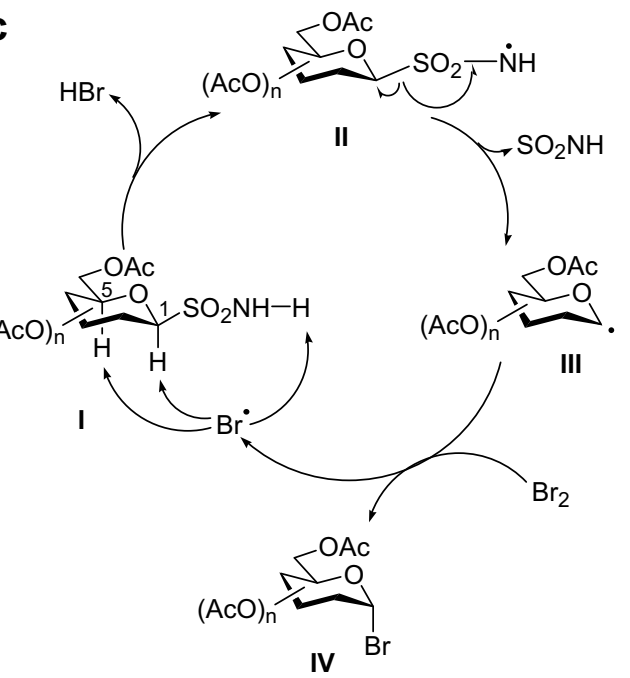

Figure 1. (a) Relative radical stabilization factors taken from Table 2 for glucopyranosyl sulfones and (b) anhydro-aldonic acid derivatives; (c) proposed reactivity pattern for the radical-mediated bromination of anomeric sulfonamides.

access to the $\mathrm{NH}_{2}$ as compared to the ring positions, but radical stabilization factors may also play a role.

In 1-bromoglucopyranosyl sulfonamide $\mathbf{2 8}$ as well as sulfone $\mathbf{2 4}$ some nucleophilic substitutions, reactions analogous to those carried out easily ${ }^{1-3,35,36}$ with $O$-peracyl derivatives of (D-glyco-hept2-ulopyranosyl bromide)onamides (Chart 1, A) were attempted. Contrary to these transformations, reactions either with azide or thiocyanate ions failed: either no change (28 and AgSCN or $\mathrm{NH}_{4} \mathrm{SCN}, \mathrm{CH}_{3} \mathrm{NO}_{2}, 80^{\circ} \mathrm{C}, \mathrm{Ar}$ atm; 28 and $\mathrm{NaN}_{3}$, DMF, rt; 24 and $\mathrm{NaN}_{3}$, acetone, $\mathrm{LiN}_{3}, \mathrm{DMF}$, or acetone, each at rt) or decomposition (24 and $\mathrm{NaN}_{3}$, DMF, rt; AgOTF, TMSN $3, \mathrm{CH}_{2} \mathrm{Cl}_{2}, \mathrm{rt}$ ) of the starting material could be observed.

The prepared 1-thioglucoside $\mathbf{5}$ and glucosyl sulfonyl derivatives $\mathbf{1 1}$ and $\mathbf{1 4}$ were deprotected as shown in Scheme 1 to give compounds 6, 12 and 15, respectively. These compounds were tested as inhibitors of RMGPb (Table 3 ) in the way described earlier. ${ }^{3}$ While 1 -thio- $\beta$-D-glucopyranose (entry 1 ) is a moderate inhibitor, its glycosides are either non-inhibitory (entries 2 and $\widehat{3}$ ) or only slightly more efficient (entry 4 ). The latter effect may be due to interactions of the bulky aglycon in the so called $\beta$-channel of the enzyme. ${ }^{7,8}$ Sulfon $\mathbf{1 2}$ (entry 5) and sulfonamide $\mathbf{1 5}$ (entry 6 ) were inactive. Comparing the latter result to the effect of the 2,6-anhydro-aldonamide in entry 7 shows that introduction of the tetrahedral sulfonyl moiety in the $\beta$-anomeric position of $D-$ glucose is not a useful modification with respect to inhibition of RMGPb.

In conclusion, a synthetic sequence has been elaborated for the preparation of $\mathrm{N}$-unsubstituted $\beta$-D-glycopyranosyl-1-C-sulfonamides, which circumvents the unavailability of anomeric sulfonyl
Table 3

Inhibition of RMGP $b$ by selected 1-thio derivatives of D-glucose

6

chlorides. Further functionalization at $\mathrm{C}-1$ of these sulfonamides by radical-mediated bromination, although possible, has proven limited because of formation of the corresponding glycosyl bromides and 5-C-bromides as accompanying products. 1-Bromoglycosyl sulfones and sulfonamides seem to be unreactive in nucleophilic substitutions. Appending the tetrahedral sulfonyl moiety to the anomeric centre of D-glucose results in a loss of activity against RMGPb.

\section{Experimental}

\subsection{General methods}

Melting points were measured in open capillary tubes or on a Kofler hot-stage and are uncorrected. Optical rotations were determined on a Perkin-Elmer 241 polarimeter at room temperature. NMR spectra were recorded with Bruker WP 360 SY $(360 / 90 \mathrm{MHz}$ for $\left.{ }^{1} \mathrm{H} /{ }^{13 \mathrm{C}}\right)$ and Varian UNITYINOVA $400 \mathrm{WB}(400 / 100 \mathrm{MHz}$ for ${ }^{1} \mathrm{H} /{ }^{13} \mathrm{C}$ ) spectrometers. Chemical shifts are referenced to $\mathrm{Me}_{4} \mathrm{Si}$ as the internal reference $(1 \mathrm{H})$ or to the residual solvent signal $\left({ }^{13} \mathrm{C}\right)$. ESIMS were recorded with a Bruker micrOTOF-Q instrument. Thin-layer chromatography (TLC) was carried out on aluminium sheets coated with Silica Gel $60 \mathrm{~F}_{254}$ (Merck). TLC plates were in-

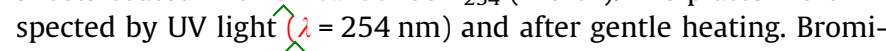
nated compounds were visualized on TLC plates by spraying first a $0.1 \% \mathrm{w} / \mathrm{v}$ fluorescein soln in absolute $\mathrm{MeOH}$, then a $1: 1$ mixture

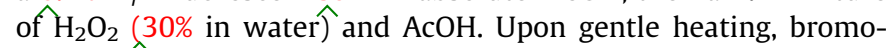
compounds gave pink-coloured spots. Silica gel column chromatography was performed with Silica Gel Si $60(40-63 \mu \mathrm{m})$ purchased from Merck (Darmstadt, Germany). Organic solutions were dried over anhydrous $\mathrm{MgSO}_{4}$, and concentrated at diminished pressure at $40-50{ }^{\circ} \mathrm{C}$ (water bath). 


\section{2. $2,3,4,6$-Tetra-O-acetyl-1- $S$-acetyl-1-thio- $\beta$-D-glucopyranose (adapted from $\left.{ }^{14}\right)(2)$}

1,2,3,4,6-Penta- $O$-acetyl- $\beta$-D-glucopyranose $\quad(\mathbf{1}, \quad 1.00 \mathrm{~g}, \quad 2.56$ $\mathrm{mmol})$ was dissolved in dry $\mathrm{CH}_{2} \mathrm{Cl}_{2}(10 \mathrm{~mL})$. To the soln, thioacetic acid $(0.28 \mathrm{~mL}, 3.84 \mathrm{mmol})$ and $\mathrm{BF}_{3} \mathrm{OEt}_{2}(0.54 \mathrm{~mL}, 5.12 \mathrm{mmol})$ were added at $0{ }^{\circ} \mathrm{C}$. The mixture was then stirred at $\mathrm{xt}$ for $1 \mathrm{~d}$, and after completion of the reaction (TLC, 95:5 $\mathrm{CH}_{2} \mathrm{Cl}_{2}$-acetone) it was diluted with $\mathrm{CH}_{2} \mathrm{Cl}_{2}(10 \mathrm{~mL})$, washed with satd $\mathrm{NaHCO}_{3}$ $(2 \times 10 \mathrm{~mL})$ and water $(1 \times 10 \mathrm{~mL})$. After drying the solvent was removed under diminished pressure. The crude product was crystallized from EtOH to give $0.97 \mathrm{~g}(93 \%)$ of 2 as a white crystalline product. Mp: $119-120^{\circ} \mathrm{C}$, lit. $.^{15} \mathrm{mp}: 118^{\circ} \mathrm{C} ;[\alpha]_{\mathrm{D}}+29($ c 0.38 , $\left.\mathrm{CHCl}_{3}\right)$, (lit. ${ }^{15}[\alpha]_{\mathrm{D}}+11$ (c 1.49, $\left.\left.\mathrm{CHCl}_{3}\right)\right)$; ${ }^{1} \mathrm{H}$ NMR $\left(\mathrm{CDCl}_{3}\right.$, $360 \mathrm{MHz}$ ): $\delta(\mathrm{ppm}) 5.27,5.12,5.10$ (pseudo $\mathrm{t}, 3 \mathrm{H}, J \sim 9.2 \mathrm{~Hz}$ in each, H-2, H-3, H-4), 5.26 (d, 1H, J1,2 $9.2 \mathrm{~Hz}, \mathrm{H}-1$ ), 4.24 (dd, $1 \mathrm{H}$,

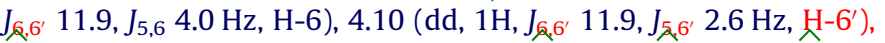
3.86 (ddd, $\left.1 \mathrm{H}, J_{4,5} 9.2, J_{5,6} 4.0, J_{\Omega^{\prime} 6^{\prime}} 2.6 \mathrm{~Hz}, \mathrm{H}-5\right), 2.39$ (s, 3H, $\mathrm{SCOCH}_{3}$ ), $2.08,2.03,2.02,2.01\left(12 \mathrm{H}, 4 \mathrm{~s}, 4 \times \mathrm{OCOCH}_{3}\right) ;{ }^{13} \mathrm{C} \mathrm{NMR}\left(\mathrm{CDCl}_{3}\right.$, $90 \mathrm{MHz}): \delta(\mathrm{ppm}) 191.8\left(\mathrm{SCOCH}_{3}\right), 170.5,169.2,169.3$ (2) (CO), 80.1 (C-1), 76.3, 73.8, 68.9, 67.8 (C-2 to C-5), 61.6 (C-6), 30.7 $\left(\mathrm{SCOCH}_{3}\right) 20.6,20.5(3)\left(\mathrm{CH}_{3}\right)$.

\subsection{Sodium $2,3,4,6$-tetra- $O$-acetyl- $\beta$-D-glucopyranosyl-1-C- sulfonate (adapted from $\left.{ }^{14}\right)(4)$}

(a) Compound 2 ( $0.50 \mathrm{~g}, 1.23 \mathrm{mmol})$ was suspended in glacial acetic acid $(10 \mathrm{~mL})$, and Oxone ${ }^{\circledR}(1.89 \mathrm{~g}, 3.07 \mathrm{mmol})$ and $\mathrm{NaOAc}$ $(1.34 \mathrm{~g}, 12.3 \mathrm{mmol})$ were added. The mixture was stirred at rt until TLC (4:1 $\left.\mathrm{CH}_{2} \mathrm{Cl}_{2}-\mathrm{MeOH}\right)$ showed complete transformation of the starting material. After filtration the solvent was removed under diminished pressure. The obtained crude product was purified by column chromatography (eluent, $4: 1 \mathrm{CHCl}_{3}-\mathrm{MeOH}$ ) to give $0.42 \mathrm{~g}$ $(70 \%)$ of 4 as a white crystalline product. $\mathrm{Mp}: 218-220^{\circ} \mathrm{C} ;[\alpha]_{\mathrm{D}}$ -59 (c $\left.0.23, \mathrm{H}_{2} \mathrm{O}\right) ;{ }^{1} \mathrm{H}$ NMR $\left(\mathrm{D}_{2} \mathrm{O}, 360 \mathrm{MHz}\right): \delta(\mathrm{ppm}) 5.43,5.37$, 5.17 (pseudo t, $3 \mathrm{H}, J \sim 9.4 \mathrm{~Hz}$ in each, $\mathrm{H}-2, \mathrm{H}-3, \mathrm{H}-4), 4.57$ (d, $1 \mathrm{H}$, $J_{1,2} 9.4 \mathrm{~Hz}, \mathrm{H}-1$ ), 4.43 (dd, $\left.1 \mathrm{H}, J_{6,6^{\prime}} 11.7, J_{5,6} 2.1 \mathrm{~Hz}, \mathrm{H}-6\right), 4.27$ (dd, $\left.1 \mathrm{H}, J_{6,6^{\prime}} 11.7, J_{5,6^{\prime}} 1.0 \mathrm{~Hz}, \mathrm{H}-6^{\prime}\right), 4.14$ (ddd, $1 \mathrm{H}, J_{4,5} 9.4, J_{5,6} 2.1, J_{5,6^{\prime}}$ $1.0 \mathrm{~Hz}, \mathrm{H}-5), 2.15,2.12,2.11,2.09\left(12 \mathrm{H}, 4 \mathrm{~s}, 4 \times \mathrm{OCOCH}_{3}\right) ;{ }^{3} \mathrm{C}$ NMR $\left(\mathrm{D}_{2} \mathrm{O}, 90 \mathrm{MHz}\right): \delta(\mathrm{ppm}) 174.2,173 . \widehat{6}, 173.2,172.8(\mathrm{CO})$, 85.5 (C-1), 75.7, 74.7, 69.5, 68.5 (C-2 to C-5), 62.5 (C-6), 21.9, 20.8, 20.7, $20.6\left(\mathrm{CH}_{3}\right)$; ESI MS calcd for $\mathrm{C}_{14} \mathrm{H}_{19} \mathrm{O}_{12} \mathrm{SNa} 434.354$, found $457.018(\mathrm{M}+\mathrm{Na})$.

(b) Also prepared from 2,3,4,6-tetra- $O$-acetyl-1-thio- $\beta$-D-glucopyranose $^{17}(3,0.50 \mathrm{~g}, 1.31 \mathrm{mmol})$ by using the same procedure. Yield: $0.52 \mathrm{~g}(87 \%)$.

\subsection{Methyl 3-(2,3,4,6-tetra-O-acetyl- $\beta$-D-} glucopyranosylthio)propanoate (5)

Compound 1 (5.00 g, $12.8 \mathrm{mmol}$ ) was dissolved in dry $\mathrm{CH}_{2} \mathrm{Cl}_{2}$ $(30 \mathrm{~mL})$, then methyl 3-sulfanylpropanoate $(2.1 \mathrm{~mL}, 19.2 \mathrm{mmol}$ ) and $\mathrm{BF}_{3} \cdot \mathrm{OEt}_{2}(2.7 \mathrm{~mL}, 25.6 \mathrm{mmol})$ were added at $0^{\circ} \mathrm{C}$. The soln was stirred overnight $(\sim 16 \mathrm{~h})$ at rt, (TLC, 1:2 EtOAc-hexane). After complete transformation of the starting material, the mixture was diluted with $\mathrm{CH}_{2} \mathrm{Cl}_{2}$ and washed with satd $\mathrm{NaHCO}_{3}(2 \times 15 \mathrm{~mL})$ and water $(1 \times 15 \mathrm{~mL})$, and then dried. The solvent was removed under diminished pressure and the crude product crystallized from EtOH to give $2.50 \mathrm{~g}$ (43\%) of 5 as white needles. Mp: $86-87^{\circ} \mathrm{C}$ (lit. $^{22}$ mp: $\left.88-89{ }^{\circ} \mathrm{C}\right) ;\left[\alpha \hat{\mathrm{D}}_{\mathrm{D}}-64\right.$ (c 0.26, $\mathrm{CHCl}_{3}\left(\mathrm{lit}^{22}[\alpha]_{\mathrm{D}}-32\right.$ (c 1.00, $\left.\mathrm{CHCl}_{3}\right)$ ); ${ }^{1} \mathrm{H} \mathrm{NMR}\left(\mathrm{CDCl}_{3}, 360 \mathrm{MHz}\right): \delta(\mathrm{ppm})$ 5.23, 5.03, 5.01 (pseudo t, $3 \mathrm{H}, J \sim 9.2 \mathrm{~Hz}$ in each, H-2, H-3, H-4), 4.56 (d, $1 \mathrm{H}, J_{1,2} 10.6 \mathrm{~Hz}$, $\mathrm{H}-1$ ), 4.23 (dd, $\left.1 \mathrm{H}, J_{6,6}, 11.9, J_{5,6} 5.3 \mathrm{~Hz}, \mathrm{H}-6\right), 4.14$ (dd, $1 \mathrm{H}, J_{6,6}, 11.9$, $\left.J_{5,6^{\prime}} 2.6 \mathrm{~Hz}, \mathrm{H}-6^{\prime}\right), 3.74$ (ddd, $1 \mathrm{H}, J_{4,5} 9.2, J_{5,6} 5.3, J_{5,6^{\prime}} 2.6 \mathrm{~Hz}, \mathrm{H}-5$ ), $3.69\left(\mathrm{~s}, 3 \mathrm{H}, \mathrm{OCH}_{3}\right), 2.99\left(\mathrm{dt}, 1 \mathrm{H}, J 7.9, J 6.6 \mathrm{~Hz}, \mathrm{CH}_{2}\right), 2.88(\mathrm{dt}, 1 \mathrm{H}$, $J$ 7.9, J $6.6 \mathrm{~Hz}, \mathrm{CH}_{2}$ ), $2.69\left(\mathrm{t}, 2 \mathrm{H}, J 7.9, J 6.6 \mathrm{~Hz}, \mathrm{CH}_{2}\right.$ ), 2.09, 2.06,
2.03, $2.01\left(12 \mathrm{H}, 4 \mathrm{~s}, 4 \times \mathrm{OCOCH}_{3}\right) ;{ }^{13} \mathrm{C} \mathrm{NMR}\left(\mathrm{CDCl}_{3}, 90 \mathrm{MHz}\right): \delta$ (ppm) $171.9\left(\mathrm{COOCH}_{3}\right), 170.5,169.9,169.2$ (2) (CO), 83.7 (C-1), 75.7, 73.6, 69.5, 68.1 (C-2 to C-5), 61.9 (C-6), $51.6\left(\mathrm{OCH}_{3}\right), 35.1$, $25.2\left(\mathrm{CH}_{2}\right), 20.5,20.4(2), 20.3\left(\mathrm{CH}_{3}\right)$. Anal. C्रalcd for $\mathrm{C}_{18} \mathrm{H}_{26} \mathrm{O}_{11} \mathrm{~S}_{1}$ (450.47): C., 47.99; H, 5.82. Found: C., 47.86; H, 5.66.

\subsection{Methyl 3-( $\beta$-D-glucopyranosylthio)propanoate (6)}

Thioglucoside 5 ( $0.18 \mathrm{~g}, 0.41 \mathrm{mmol})$ was dissolved in dry $\mathrm{MeOH}$ (5 $\mathrm{mL})$, and $\mathrm{NaOMe}(\sim 1 \mathrm{M}$ in $\mathrm{MeOH})$ was added. The reaction mixture was kept at th until completion of the transformation (TLC, $1: 1$ $\left.\mathrm{CHCl}_{3}-\mathrm{MeOH}\right)$. Amberlyst $15\left(\mathrm{H}^{+}\right.$form) was then added to remove sodium ions, the resin was filtered off, and the solvent was evaporated to give $0.11 \mathrm{~g}(96 \%)$ of $\mathbf{6}$ as a colourless oil. $R_{\mathrm{f}}=0.73(1: 1$ $\left.\mathrm{CHCl}_{3}-\mathrm{MeOH}\right) ;[\alpha]_{\mathrm{D}}-74\left(\right.$ c 0.19, $\left.\mathrm{H}_{2} \mathrm{O}\right) ;{ }^{1} \mathrm{H}$ NMR $\left(\mathrm{D}_{2} \mathrm{O}, 360 \mathrm{MHz}\right)$ : $\delta(\mathrm{ppm}) 4.51$ (dd, $\left.1 \mathrm{H}, J_{\bar{\gamma}, 6^{\prime}} 13.2, J_{5,6} 4.0 \mathrm{~Hz}, \mathrm{H}-6\right), 3.85$ (dd, $1 \mathrm{H}, J_{\mathbf{C}^{6} 6^{\prime}}$

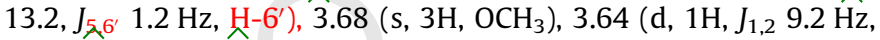
$\mathrm{H}-1$ ), 3.44-3.23 (m, 3H, H-2, H-3, H-4), 3.26 (ddd, $1 \mathrm{H}, J_{4,5} 9.2, J_{5,6}$ 4.0, $\left.J_{\text {攵 } 6^{\prime}} 1.2 \mathrm{~Hz}, \mathrm{H}-5\right), 2.99$ (quintet, $\left.1 \mathrm{H}, J 7.9, J 6.6 \mathrm{~Hz}, \mathrm{CH}_{2}\right), 2.93$ (quintet, $\left.1 \mathrm{H}, J 7.9, J 6.6 \mathrm{~Hz}, \mathrm{CH}_{2}\right), 2.76-2.74\left(\mathrm{~m}, 2 \mathrm{H}, \mathrm{CH}_{2}\right) ;{ }^{13} \mathrm{C}$ NMR $\left(\mathrm{D}_{2} \mathrm{O}, 90 \mathrm{MHz}\right) . \delta(\mathrm{ppm}) 175.7\left(\mathrm{COOCH}_{3}\right), 86.5(\mathrm{C}-1), 80.4$, 77.4, 72.7, 70.0, (C-2 to $\mathrm{C}-5), 61.5$ (C-6), $53.5\left(\mathrm{OCH}_{3}\right), 35.5,25.7$ $\left(\mathrm{CH}_{2}\right)$. Anal. Calcd for $\mathrm{C}_{10} \mathrm{H}_{18} \mathrm{O}_{7} \mathrm{~S}_{1}(282.31)$ : $\mathrm{C}, 42.55 ; \mathrm{H}, 6.43$. Found: C. $42.50 ; \mathrm{H}, 6.48$.

\subsection{Methyl 3-(2,3,4,6-tetra- 0 -acetyl- $\beta$-D- glucopyranosylthio)propanoate $S$-oxide (10)}

Thioglucoside $5(0.1 \mathrm{~g}, 0.22 \mathrm{mmol})$ was dissolved in $\mathrm{MeOH}$ (3 mL). To this soln $\mathrm{Na}_{2} \mathrm{WO}_{4}(6.5 \mathrm{mg}, 0.02 \mathrm{mmol})$ and $\mathrm{H}_{2} \mathrm{O}_{2}$ $(0.1 \mathrm{~mL}, 3.30 \mathrm{mmol})$ were added in small portions. The mixture was stirred at rt until completion of the transformation (TLC, $1: 1$ EtOAc-hexane). After filtration the solvent was removed under diminished pressure. The obtained oil was purified by column chromatography (2:1 EtOAc-hexane) to give $0.04 \mathrm{~g}$ (39\%) of $\mathbf{1 0}$ as a white powder. Mp: $115-117{ }^{\circ} \mathrm{C} ;[\alpha]_{\mathrm{D}}-128\left(\mathrm{c} 0.22, \mathrm{CHCl}_{3}\right) ;{ }^{1} \mathrm{H}$ $\operatorname{NMR}\left(\mathrm{CDCl}_{3}, 360 \mathrm{MHz}\right): \delta(\mathrm{ppm}) 5.34,5.26,5.11$ (pseudo t, $3 \mathrm{H}$, $J \sim 9.2 \mathrm{~Hz}$ in each, H-2, H-3, H-4), 4.37 (d, $\left.1 \mathrm{H}, J_{1,2} 9.2 \mathrm{~Hz}, \mathrm{H}-1\right)$, 4.29 (dd, $\left.1 \mathrm{H}, J_{6,6^{\prime}} 13.2, J_{5,6} 5.3 \mathrm{~Hz}, \mathrm{H}-6\right), 4.21$ (dd, $1 \mathrm{H}, J_{6,6^{\prime}} 13.2, J_{5,6^{\prime}}$ $2.6 \mathrm{~Hz}, \mathrm{H}^{-6^{\prime}}$ ), 3.82 (ddd, $1 \mathrm{H}, J_{4,5} 9.2, J_{5,6} 5.3, J_{\text {, } 6^{\prime}} 2.6 \mathrm{~Hz}, \mathrm{H}-5$ ), 3.73 $\left(\mathrm{s}, 3 \mathrm{H}, \mathrm{OCH}_{3}\right), 3.22-3.20\left(\mathrm{~m}, 2 \mathrm{H}, \mathrm{CH}_{2}\right), 2.85\left(\mathrm{~m}, 2 \mathrm{H}, \mathrm{CH}_{2}\right), 2.09$, $2.07,2.05,2.03\left(12 \mathrm{H}, 4 \mathrm{~s}, 4 \times \mathrm{OCOCH}_{3}\right) ;{ }^{13} \mathrm{C} \mathrm{NMR}\left(\mathrm{CDCl}_{3}\right.$, $\left.90 \mathrm{MHz}): \delta(\mathrm{ppm}) 171.5 \widehat{(C O O C H}_{3}\right), 170.4,169.9,169.5,169.2$ (CO), 90.1 (C-1), 76.8, 73.0, 68.3, 67.5 (C-2 to C-5), 61.3 (C-6), $52.2\left(\mathrm{OCH}_{3}\right), 42.0,26.1\left(\mathrm{CH}_{2}\right), 20.5(2), 20.4(2)\left(\mathrm{CH}_{3}\right)$. Anal. Calcd for $\mathrm{C}_{18} \mathrm{H}_{26} \mathrm{O}_{12} \mathrm{~S}_{1}$ (466.46): C, 46.35; H, 5.62. Found: $\mathrm{C}, 46.1 \hat{5} ; \mathrm{H}$, 5.55 .

\subsection{Methyl 3-(2,3,4,6-tetra-O-acetyl- $\beta$-D-} glucopyranosylthio)propanoate $S, S$-dioxide (11)

(a) Thioglucoside $5(0.1 \mathrm{~g}, 0.22 \mathrm{mmol})$ was dissolved in glacial acetic acid $(3 \mathrm{~mL})$. To this soln, Oxone ${ }^{\circledR}(0.19 \mathrm{~g}, 0.33 \mathrm{mmol})$ and NaOAc $(0.14 \mathrm{~g}, 2.20 \mathrm{mmol})$ were added. The mixture was stirred at rt until complete transformation of the starting material (TLC, $1: \hat{\Upsilon}$ EtOAc-hexane). After filtration it was diluted with water $(10 \mathrm{~mL})$ and washed with $\mathrm{CH}_{2} \mathrm{Cl}_{2}(3 \times 5 \mathrm{~mL})$. The organic phase was washed with satd $\mathrm{NaHCO}_{3}(2 \times 5 \mathrm{~mL})$ and water $(1 \times 5 \mathrm{~mL})$, dried, and the solvent removed under diminished pressure. The crude product was recrystallized from $\mathrm{Et}_{2} \mathrm{O}$ to give $0.07 \mathrm{~g}$ (70\%) of 11.

(b) Thioglucoside 5 ( $0.1 \mathrm{~g}, 0.22 \mathrm{mmol})$ was suspended in $\mathrm{CH}_{2} \mathrm{Cl}_{2}$ $(3 \mathrm{~mL})$, and $\mathrm{NaHCO}_{3}(0.19 \mathrm{~g}, 2.20 \mathrm{mmol})$ and $m$ CPBA $(0.22 \mathrm{~g}$, $15.8 \mathrm{mmol}, \sim 70 \%)$ were added in five portions at $0{ }^{\circ} \mathrm{C}$. The mixture was stirred at rt until complete transformation of the starting material (TLC, $\widehat{1} 1$ EtOAc-hexane). After filtration it was diluted 
with water $(10 \mathrm{~mL})$ and washed with $\mathrm{CH}_{2} \mathrm{Cl}_{2}(2 \times 5 \mathrm{~mL})$. The organic phase was washed with $\mathrm{NaCl}(1 \times 5 \mathrm{~mL})$, satd $\mathrm{NaHCO}_{3}$ $(2 \times 5 \mathrm{~mL})$ and water $(1 \times 5 \mathrm{~mL})$, dried, and the solvent removed under diminished pressure. The crude product was recrystallized from $\mathrm{Et}_{2} \mathrm{O}$ to yield $0.08 \mathrm{~g}(72 \%)$ of $\mathbf{1 1}$ as white crystalline product. Mp: $146-147^{\circ} \mathrm{C} ;[\alpha]_{\mathrm{D}}-36\left(\mathrm{c} 0.22, \mathrm{CHCl}_{3}\right) ;{ }^{1} \mathrm{H} \mathrm{NMR}\left(\mathrm{CDCl}_{3}\right.$, $360 \mathrm{MHz}$ ): $\delta(\mathrm{ppm}) 5.52,5.33,5.14$ (pseudo $\mathrm{t}, 3 \mathrm{H}, J \sim 9.3 \mathrm{~Hz}$ in each, H-2, H-3, H-4), 4.56 (d, $\left.1 \mathrm{H}, J_{1,2} 9.2 \mathrm{~Hz}, \mathrm{H}-1\right), 4.28$ (dd, $1 \mathrm{H}$,

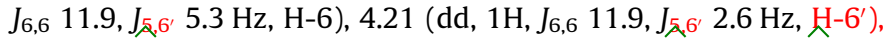
3.88 (ddd, 1H, J4,5 9.3, J5,6 5.3, $\left.J_{\delta^{2}, 6^{\prime}} 2.6 \mathrm{~Hz}, \mathrm{H}-5\right), 3.75(\mathrm{~s}, 3 \mathrm{H}$, $\left.\mathrm{OCH}_{3}\right), 3.60\left(\mathrm{dt}, 1 \mathrm{H}, J 7.9,6.6 \mathrm{~Hz}, \mathrm{CH}_{2}\right), 3.41(\mathrm{dt}, 1 \mathrm{H}, J 7.9,6.6 \mathrm{~Hz}$, $\left.\mathrm{CH}_{2}\right), 2.89\left(\mathrm{~m}, 2 \mathrm{H}, \mathrm{CH}_{2}\right), 2.10,2.05(2), 2.03\left(12 \mathrm{H}, 4 \mathrm{~s}, 4 \times \mathrm{OCOCH}_{3}\right)$; ${ }^{13} \mathrm{C} \mathrm{NMR}\left(\mathrm{CDCl}_{3}, 90 \mathrm{MHz}\right): \delta(\mathrm{ppm}) 170.5\left(\mathrm{COOCH}_{3}\right), 170.3,169.9$, 169.2, 169.1 (CO), 87.9 (C-1), 76.6, 72.9, 67.2, 66.2 (C-2 to C-5), 61.2 (C-6), $52.3\left(\mathrm{OCH}_{3}\right), 45.3,26.2\left(\mathrm{CH}_{2}\right), 20.4(2), 20.3(2)\left(\mathrm{CH}_{3}\right)$. Anal. Calcd for $\mathrm{C}_{18} \mathrm{H}_{26} \mathrm{O}_{13} \mathrm{~S}_{1}$ (482.46): . 44.81 ; H, 5.43. Found: $\mathcal{X}_{\text {, }}$ 44.78; H, 5.40 .

\subsection{Methyl 3-( $\beta$-D-glucopyranosylthio)propanoate $S, S$-dioxide} (12)

Sulfone $\mathbf{1 1}(0.1 \mathrm{~g}, 0.21 \mathrm{mmol})$ was suspended in a mixture of dry $\mathrm{MeOH}(3 \mathrm{~mL})$ and dry $\mathrm{CHCl}_{3}(2 \mathrm{~mL})$. To this soln two drops of $\mathrm{AcCl}$ were added. The mixture was kept at rt until completion of the transformation ( one week, TLC, $4: 1 \mathrm{CHCl}_{3}-\mathrm{MeOH}$ ). It was then neutralized with solid $\mathrm{NaHCO}_{3}$ and the solvent evaporated after filtration. The crude oil was purified by column chromatography $(4: 1$ $\left.\mathrm{CHCl}_{3}-\mathrm{MeOH}\right)$ to give $0.05 \mathrm{~g}$ (75\%) of $\mathbf{1 2}$ as a colourless oil. $R_{\mathrm{f}}=0.37$ $\left(4: 1 \widehat{C}^{-1 C l} l_{3}-\mathrm{MeOH}\right) ;[\alpha]_{\mathrm{D}}-53\left(c \quad 0.26, \mathrm{H}_{2} \mathrm{O}\right) ;{ }^{1} \mathrm{H}$ NMR $\left(\mathrm{D}_{2} \mathrm{O}\right.$, $360 \mathrm{MHz}$ ): $\widehat{\delta}(\mathrm{ppm}) 4.68$ (d, $\left.1 \mathrm{H}, J_{1,2} 9.2 \mathrm{~Hz}, \mathrm{H}-1\right), 3.90$ (dd, $1 \mathrm{H}, J_{6,6^{\prime}}$ 13.2, $\left.J_{5,6} 5.3 \mathrm{~Hz}, \mathrm{H}-6\right), 3.85\left(\mathrm{~s}, 3 \mathrm{H}, \mathrm{OCH}_{3}\right), 3.83\left(\mathrm{dd}, 1 \mathrm{H}, J_{6,6^{\prime}} 13.2\right.$, $\left.J_{5,6^{\prime}} 2.6 \mathrm{~Hz}, \mathrm{H}-6^{\prime}\right), 3.78-3.36\left(\mathrm{~m}, 6 \mathrm{H}, \mathrm{H}-2, \mathrm{H}-3, \mathrm{H}-4, \mathrm{H}-5, \mathrm{CH}_{2}\right)$, 2.98-2.92 ( $\left.\mathrm{m}, 2 \mathrm{H}, \mathrm{CH}_{2}\right) ;{ }^{13} \mathrm{C}$ NMR $\left(\mathrm{D}_{2} \mathrm{O}, 90 \mathrm{MHz}\right): \delta(\mathrm{ppm}) 173.8$ $\left(\mathrm{COOCH}_{3}\right), 90.0(\mathrm{C}-1), 81.3,77.2,69.2,69.1,(\mathrm{C}-2$ to $\mathrm{C}-5), 61.0(\mathrm{C}-$ 6), $53.4\left(\mathrm{OCH}_{3}\right), 47.1,27.1\left(\mathrm{CH}_{2}\right)$. Anal. Calcd for $\mathrm{C}_{10} \mathrm{H}_{18} \mathrm{O}_{9} \mathrm{~S}_{1}$

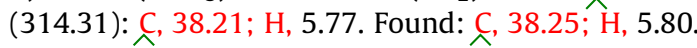

\subsection{Mixed salt of $2,3,4,6$-tetra- $O$-acetyl- $\beta$-D-glucopyranosyl-1-C- sulfinic acid (13)}

Sulfone $11(0.1 \mathrm{~g}, 0.21 \mathrm{mmol})$ was dissolved in freshly distilled dry $\mathrm{CHCl}_{3}(10 \mathrm{~mL})$, and DBU $(30 \mu \mathrm{L}, 0.21 \mathrm{mmol})$ was added. The mixture was stirred at rt until complete transformation of the starting material (TLC, 7:3 $\mathrm{CHCl}_{3}-\mathrm{MeOH}$ ). The solvent was evaporated and the crude oil was purified by column chromatography $\left(7: 3 \mathrm{CHCl}_{3}-\mathrm{MeOH}\right)$ to give $0.06 \mathrm{~g}$ of $\mathbf{1 3}$ as a white crystalline product. ${ }^{1} \mathrm{H}$ NMR $\left(\mathrm{D}_{2} \mathrm{O}, 360 \mathrm{MHz}\right): \delta(\mathrm{ppm})$ 5.36, 5.29, 5.08 (pseudo $\mathrm{t}$, $3 \mathrm{H}, \mathrm{J} \sim 9.2 \mathrm{~Hz}$ in each, H-2, H-3, H-4), 4.38 (dd, $1 \mathrm{H}, J_{6,6} 11.9, J_{5,6}$ $2.6 \mathrm{~Hz}, \mathrm{H}-6$ ), 4.27 (dd, $1 \mathrm{H}, J_{6,6^{\prime}} 11.9, J_{5,6^{\prime}} 1.0 \mathrm{~Hz}, \mathrm{H}^{\prime} 6^{\prime}$ ), 4.00 (ddd, $\left.1 \mathrm{H}, J_{4,5} 9.2, J_{5,6} 2.6, J_{5,6^{\prime}} 1.0 \mathrm{~Hz}, \mathrm{H}-5\right), 3.66\left(\mathrm{~d}, 1 \mathrm{H}, \mathrm{J}_{1,2} 9.2 \mathrm{~Hz}, \mathrm{H}-1\right)$, $2.10,2.05,2.04(2)\left(12 \mathrm{H}, 3 \mathrm{~s}, 4 \times \mathrm{OCOCH}_{3}\right) ;{ }^{13} \mathrm{C} \mathrm{NMR}\left(\mathrm{D}_{2} \mathrm{O}\right.$, $90 \mathrm{MHz}): \delta(\mathrm{ppm}) 174.3,173.9,173.3$ (2) (CO), 92.8 (C-1), 75.8, 75.2, 68.7, 68.5 (C-2 to C-5), 62.5 (C-6), 20.9, 20.7, $20.6(2)\left(\mathrm{CH}_{3}\right)$; Ms: $[\mathrm{M}+\mathrm{Na}]^{+} 419.059,[\mathrm{M}+\mathrm{K}]^{+}$435.035. Repeated from $1.5 \mathrm{~g}$ sulfone. Yield: $1.35 \mathrm{~g}(\sim 100 \%)$. For further preparative purposes, the crude product obtained after solvent removal was used without purification.

\subsection{2,3,4,6-Tetra-O-acetyl- $\beta$-D-glucopyranosyl-1-C- sulfonamide $(14)$}

Sulfinate $13(0.1 \mathrm{~g})$ was suspended in water $(5 \mathrm{~mL})$. To this soln, $\mathrm{H}_{2} \mathrm{NOSO}_{3} \mathrm{H}(0.08 \mathrm{~g}, 0.71 \mathrm{mmol})$ and $\mathrm{NaOAc}(0.06 \mathrm{~g}, 0.73 \mathrm{mmol})$ were added and the mixture was stirred at rt for $2 \mathrm{~h}$. After extraction with EtOAc, $(5 \times 5 \mathrm{~mL})$ the organic phase was washed with satd $\mathrm{NaHCO}_{3}(2 \times 5 \mathrm{~mL})$ and water $(5 \mathrm{~mL})$, dried and evaporated.
It was crystallized slowly to give $0.07 \mathrm{~g}$ ( $81 \%$, from the sulfone, $0.1 \mathrm{~g}$ ) of 14 as a white crystalline product. Mp: $201-204{ }^{\circ} \mathrm{C} ;[\alpha]_{\mathrm{D}}$ -20 (c $\left.0.2, \mathrm{CHCl}_{3}\right) ;{ }^{1} \mathrm{H}$ NMR $\left(\mathrm{CDCl}_{3}, 360 \mathrm{MHz}\right): \delta(\mathrm{ppm}) 5.39$, 5.31, 5.14 (pseudo t, $3 \mathrm{H}, J \sim 9.3 \mathrm{~Hz}$ in each, $\mathrm{H}-2, \mathrm{H}-3, \mathrm{H}-4$ ), 4.56 (d, $\left.1 \mathrm{H}, J_{1,2} 9.2 \mathrm{~Hz}, \mathrm{H}-1\right), 4.36$ (dd, $\left.1 \mathrm{H}, J_{6,6^{\prime}} 13.2, J_{5,6} 5.3 \mathrm{~Hz}, \mathrm{H}-6\right)$, 4.46 (dd, $\left.1 \mathrm{H}, J_{\text {风 } 6^{\prime}} 13.2, J_{\text {久 } 6^{\prime}} 1.0 \mathrm{~Hz}, \mathrm{H}^{\prime} 6^{\prime}\right), 3.93-3.86(\mathrm{~m}, 3 \mathrm{H}, \mathrm{H}-5$, $\left.\mathrm{NH}_{2}\right), 2.11,2.08,2.06,2.04\left(12 \mathrm{H}, 4 \mathrm{~s}, 4 \times \mathrm{OCOCH}_{3}\right) ;{ }^{13} \mathrm{C} \mathrm{NMR}\left(\mathrm{CDCl}_{3}\right.$, $90 \mathrm{MHz}): \delta(\mathrm{ppm}) 170.9,170.4,170.1,169.5(\mathrm{CO}), 87.0(\mathrm{C}-1), 75.9$, 72.9, 67.8, 67.5 (C-2 to C-5), 61.3 (C-6), 20.2 (2), 20.1 (2) $\left(\mathrm{CH}_{3}\right)$. Anal. Calcd for $\mathrm{C}_{14} \mathrm{H}_{21} \mathrm{O}_{11} \mathrm{NS}$ (411.39): $\mathrm{C}_{\text {, }} 40.88$; H, 5.15. Found: $\mathrm{C}_{\text {, }}$ 40.82; H, 5.05. Ms: [M+Na] ${ }^{+}$434.081. Repeated from 1.5 sulfone. Yield: $1.03 \mathrm{~g}$ ( $81 \%)$.

\subsection{1. $k^{-D-G l u c o p y r a n o s y l-1-C-s u l f o n a m i d e ~(15) ~}$}

Sulfonamide $14(0.1 \mathrm{~g}, 0.24 \mathrm{mmol})$ was suspended in dry $\mathrm{MeOH}$ ( $3 \mathrm{~mL}$ ). A few drops of $\mathrm{NH}_{4} \mathrm{OH}$ were added and the mixture was kept at rt until completion of the transformation (TLC, 4:1 $\left.\mathrm{CHCl}_{3}-\mathrm{MeOH}\right)$. Amberlyst $15\left(\mathrm{H}^{+}\right.$form) was then added to remove sodium ions, the resin was filtered off and the solvent removed under diminished pressure. The crude oil was purified by column chromatography $\left(4: 1 \mathrm{CHCl}_{3}-\mathrm{MeOH}\right)$ to give $0.05 \mathrm{~g}$ ( $\left.85 \%\right)$ of $\mathbf{1 5}$ as a colourless oil. $\hat{R}_{\mathrm{f}}=0.59\left(1: 1 \mathrm{CHCl}_{3}-\mathrm{MeOH}\right) ;[\alpha]_{\mathrm{D}} \overline{\mathrm{A}}^{34}$ (c 0.24 , $\left.\mathrm{H}_{2} \mathrm{O}\right) ;{ }^{1} \mathrm{H}$ NMR $\left(\mathrm{D}_{2} \mathrm{O}, 360 \mathrm{MHz}\right): \delta(\mathrm{ppm}) 4.46\left(\mathrm{~d}, 1 \mathrm{H}, J_{1,2} 9.2 \mathrm{~Hz}\right.$, $\mathrm{H}-1$ ), 3.91 (dd, $\left.1 \mathrm{H}, J_{\text {, } 6^{\prime}} 11.9, J_{5,6} 5.3 \mathrm{~Hz}, \mathrm{H}-6\right), 3.74-3.54(\mathrm{~m}, 4 \mathrm{H}$, $\mathrm{H}-2, \mathrm{H}-3$ or $\left.\mathrm{H}-4, \mathrm{H}-5, \mathrm{H}-6^{\prime}\right) 3.43(\mathrm{t}, 1 \mathrm{H}, J$ 9.2, J $9.2 \mathrm{~Hz}, \mathrm{H}-4$ or $\mathrm{H}-3)$; ${ }^{13} \mathrm{C}$ NMR $\left(\mathrm{D}_{2} \mathrm{O}, 90 \mathrm{MHz}\right): \delta(\mathrm{ppm}) 90.5(\mathrm{C}-1), 80.9,77.1,70.8$, 69.5, (C-2 to C-5), 61.2 (C-6); Anal. C्रalcd for $\mathrm{C}_{6} \mathrm{H}_{13} \mathrm{O}_{7} \mathrm{~S}_{1}$ (243.24): C. 29.60; H, 5.39. Found: C्र, 29.65; H, 5.36.

\subsection{Methyl 3-(2,3,4,6-tetra-O-acetyl- $\alpha-D-$} galactopyranosylthio)propanoate (18)

1,2,3,4,6-Penta- $O$-acetyl- $\beta$-D-galactopyranose (16, $5.0 \mathrm{~g}, 12.8$ mmol) was dissolved in dry $\mathrm{CH}_{2} \mathrm{Cl}_{2}$ (30 $\mathrm{mL}$ ), and methyl 3-sulfanylpropanoate $\left(2.1 \mathrm{~mL}, 19.2 \mathrm{mmol}\right.$ ) and $\mathrm{BF}_{3} \cdot \mathrm{OEt}_{2}(2.7 \mathrm{~mL}, 25.6 \mathrm{mmol})$ were added at $0{ }^{\circ} \mathrm{C}$. The s्रoln was stirred overnight $(\sim 16 \mathrm{~h})$ at $\mathrm{rt}$ (TLC, 1:2 EtOAc-hexane). After complete transformation of the starting material the mixture was diluted with $\mathrm{CH}_{2} \mathrm{Cl}_{2}$ and washed with satd $\mathrm{NaHCO}_{3}(2 \times 15 \mathrm{~mL})$ and water $(1 \times 15 \mathrm{~mL})$, dried, and the solvent removed under diminished pressure. The crude product was purified by silica gel column chromatography $(1: 2$ EtOAc-hexane) EtOH to give $2.00 \mathrm{~g}$ (35\%) of $\mathbf{1 8}$ as a colourless oil. $R_{\mathrm{f}}=0.16$ (1:2 EtOAc-hexane), $[\alpha]_{\mathrm{D}}+496$ (c $\left.0.34, \mathrm{CHCl}_{3}\right) ;{ }^{1} \mathrm{H}$

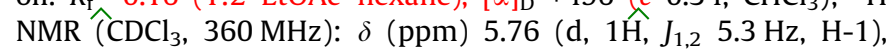
$5.45\left(\mathrm{dd}, 1 \mathrm{H}, J_{3,4} 2.6, J_{4,5} 1.0 \mathrm{~Hz}, \mathrm{H}-4\right), 5.26\left(\mathrm{dd}, 1 \mathrm{H}, J_{2,3} 10.6, J_{1,2}\right.$ $5.3 \mathrm{~Hz}, \mathrm{H}-2$ ), 5.18 (dd, $1 \mathrm{H}, J_{2,3} 10.6, J_{3,4} 2.6 \mathrm{~Hz}, \mathrm{H}-3$ ), 4.58 (t, $1 \mathrm{H}$, $\left.J_{5,6} 6.6, J_{5,6^{\prime}} 5.8 \mathrm{~Hz}, \mathrm{H}-5\right), 4.15-4.09\left(\mathrm{~m}, 2 \mathrm{H}, \mathrm{H}-6, \mathrm{H}^{\prime} 6^{\prime}\right), 3.71(\mathrm{~s}, 3 \mathrm{H}$, $\left.\mathrm{OCH}_{3}\right), 2.88\left(\mathrm{dt}, 1 \mathrm{H}, J 7.9, J 6.6 \mathrm{~Hz}, \mathrm{CH}_{2}\right), 2.78(\mathrm{dt}, 1 \mathrm{H}, J 7.9, J$ $\left.6.6 \mathrm{~Hz}, \mathrm{CH}_{2}\right), 2.68-2.64\left(\mathrm{~m}, 2 \mathrm{H}, \mathrm{CH}_{2}\right), 2.15,2.06(2), 1.96(12 \mathrm{H}, 3 \mathrm{~s}$, $\left.4 \times \mathrm{OCOCH}_{3}\right) ;{ }^{13} \mathrm{C} \mathrm{NMR}\left(\mathrm{CDCl}_{3}, 90 \mathrm{MHz}\right): \delta(\mathrm{ppm}) 171.8\left(\mathrm{COOCH}_{3}\right)$, $170.3,170.1(2), 169.7$ (CO), $89.9(\mathrm{C}-1), 67.9,67.8,67.7,66.6(\mathrm{C}-2$ to C-5), $61.8(\mathrm{C}-6), 51.7\left(\mathrm{OCH}_{3}\right), 34.4,25.1\left(\mathrm{CH}_{2}\right), 20.6,20.5(3)\left(\mathrm{CH}_{3}\right)$; Anal. Calcd for $\mathrm{C}_{18} \mathrm{H}_{26} \mathrm{O}_{11} \mathrm{~S}_{1}$ (450.47): ${ }_{\wedge}, 47.99 ; \mathrm{H}, 5.82$. Found: ${ }_{\wedge}$, $47.89 \hat{\mathrm{H}}, 5.76$.

\subsection{Methyl 3-(2,3,4,6-tetra- 0 -acetyl- $\alpha$-D- galactopyranosylthio)propanoâte $S, S$-dioxide (19)}

Thiogalactoside $18(0.5 \mathrm{~g}, 1.10 \mathrm{mmol})$ was dissolved in glacial acetic acid $(15 \mathrm{~mL})$. To this soln, Oxone ${ }^{\circledR}(0.95 \mathrm{~g}, 1.65 \mathrm{mmol})$ and NaOAc $(0.60 \mathrm{~g}, 11.0 \mathrm{mmol})$ were added. The mixture was stirred at rt until complete transformation of the starting material (TLC, $1: \hat{1}$ EtOAc-hexane). After filtration it was diluted with water

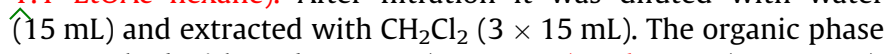
was washed with satd $\mathrm{NaHCO}_{3}(2 \times 15 \mathrm{~mL})$ and water $(1 \times 15 \mathrm{~mL})$, 
dried, and the solvent removed under diminished pressure. The crude product was purified by column chromatography $h: 1$ EtOAc-hexane) to give $0.48 \mathrm{~g}(90 \%)$ of $\mathbf{1 9}$ as a colourless oil. $R_{\mathrm{f}}=0.36$ (1:1 EtOAc-hexane), $[\alpha]_{\mathrm{D}}+33$ (c $\left.0.25, \mathrm{CHCl}_{3}\right) ;{ }^{1} \mathrm{H}$ NMR $\left(\mathrm{CDCl}_{3}, 360 \mathrm{MHz}\right): \delta(\mathrm{ppm}) 5.79\left(\mathrm{dd}, 1 \mathrm{H}, J_{2,3} 10.6, J_{3,4} 2.6 \mathrm{~Hz}, \mathrm{H}-\right.$ 3), 5.56 (dd, $\left.1 \mathrm{H}, J_{3,4} 2.6, J_{4,5} 1.0 \mathrm{~Hz}, \mathrm{H}-4\right), 5.47$ (dd, $1 \mathrm{H}, J_{2,3} 10.6$, $J_{1,2} 2.6 \mathrm{~Hz}, \mathrm{H}-2$ ), 5.36 (d, 1H, $J_{1,2} 2.6 \mathrm{~Hz}, \mathrm{H}-1$ ), 4.81 (t, 1H, J5.6 6.6, $\left.J_{\text {及, } 6^{\prime}} 5.8 \mathrm{~Hz}, \mathrm{H}-5\right), 4.19-4.06\left(\mathrm{~m}, 2 \mathrm{H}, \mathrm{H}-6, \mathrm{H}-6^{\prime}\right), 3.62\left(\mathrm{~s}, 3 \mathrm{H}, \mathrm{OCH}_{3}\right)$, 3.49-3.44 (m, $\left.2 \mathrm{H}, \mathrm{CH}_{2}\right), 2.93-2.85\left(\mathrm{~m}, 2 \mathrm{H}, \mathrm{CH}_{2}\right), 2.16,2.13,2.09$, $2.01\left(12 \mathrm{H}, 4 \mathrm{~s}, 4 \times \mathrm{OCOCH}_{3}\right) ;{ }^{13} \mathrm{C} \mathrm{NMR}\left(\mathrm{CDCl}_{3}, 90 \mathrm{MHz}\right): \delta(\mathrm{ppm})$ $170.6\left(\mathrm{COOCH}_{3}\right), 170.3,170.0,169.5,169.2(\mathrm{CO}), 84.8(\mathrm{C}-1), 71.3$, 66.9, 66.7, 65.7 (C-2 to C-5), 62.2 (C-6), $52.3\left(\mathrm{OCH}_{3}\right), 46.5,25.8$ $\left(\mathrm{CH}_{2}\right)$, 20.4, 20.2 (3) $\left(\mathrm{CH}_{3}\right)$; Anal. Calcd for $\mathrm{C}_{18} \mathrm{H}_{26} \mathrm{O}_{13} \mathrm{~S}_{1}$ (482.46):

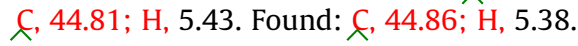

\subsection{Methyl 3-(2,3,4,6-tetra- 0 -acetyl- $\beta$-D- galactopyranosylthio)propanoate $(20)$}

A 2,3,4,6-tetra- $O$-acetyl-1-thio- $\beta$-D-galactopyranose ${ }^{25,26}$ (17, $3.0 \mathrm{~g}, 7.86 \mathrm{mmol}$ ) was dissolved in dry $\mathrm{CH}_{2} \mathrm{Cl}_{2}(50 \mathrm{~mL})$. To this soln methyl 3-bromopropanoate $(1.11 \mathrm{~mL}, 9.43 \mathrm{mmol})$ and $\mathrm{Et}_{3} \mathrm{~N}$ (1.05 mL, $9.43 \mathrm{mmol}$ ) were added. The mixture was stirred at $\mathrm{rt}$ for 3 days (TLC, 1:2 EtOAc-hexane). It was then diluted with $\mathrm{CH}_{2} \mathrm{Cl}_{2}$ and washed with satd $\mathrm{NaHCO}_{3}(2 \times 50 \mathrm{~mL})$ and water $(1 \times 50 \mathrm{~mL})$, dried, and the solvent evaporated. The crude product was purified by column chromatography (1:2 EtOAc-hexane) to give $2.10 \mathrm{~g}$ (85\%) of $\mathbf{2 0}$ (conversion is $65 \%$ ) as a yellowish oil. $R_{\mathrm{f}}=0.18$ (1:2 EtOAc-hexane); $[\alpha]_{\mathrm{D}}+169$ (c 0.42, $\left.\mathrm{CHCl}_{3}\right) ;{ }^{1} \mathrm{H}$ NMR $\left(\mathrm{CDCl}_{3}, 360 \mathrm{MHz}\right): \delta(\mathrm{ppm}) 5.40\left(\mathrm{dd}, 1 \mathrm{H}, J_{3,4} 2.6, J_{4,5} 1.0 \mathrm{~Hz}, \mathrm{H}-4\right)$ 5.23 (pseudo t, $1 \mathrm{H}, J_{2,3} 10.6, J_{1,2} 9.2 \mathrm{~Hz}, \mathrm{H}-2$ ), 5.05 (dd, $1 \mathrm{H}, J_{2,3}$ 10.6, $\left.J_{3,4} 2.6 \mathrm{~Hz}, \mathrm{H}-3\right) 4.54$ (d, $\left.1 \mathrm{H}, J_{1,2} 9.2 \mathrm{~Hz}, \mathrm{H}-1\right), 4.16$ (dd, $1 \mathrm{H}$,

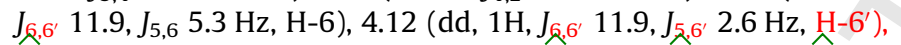
$3.95\left(\mathrm{t}, 1 \mathrm{H}, J_{5.6} 6.6, J_{5,6^{\prime}} 5.8 \mathrm{~Hz}, \mathrm{H}-5\right), 3.70\left(\mathrm{~s}, 3 \mathrm{H}, \mathrm{OCH}_{3}\right), 3.02(\mathrm{dt}$, $\left.1 \mathrm{H}, J \mathrm{~T} .9, J 6.6 \mathrm{~Hz}, \mathrm{CH}_{2}\right), 2.93\left(\mathrm{dt}, 1 \mathrm{H}, J 7.9, J 6.6 \mathrm{~Hz}, \mathrm{CH}_{2}\right), 2.72-$ $2.68\left(\mathrm{~m}, 2 \mathrm{H}, \mathrm{CH}_{2}\right), 2.16,2.05(2), 1.98\left(12 \mathrm{H}, 3 \mathrm{~s}, 4 \mathrm{OCOCH}_{3}\right) ;{ }^{13} \mathrm{C}$ NMR $\left(\mathrm{CDCl}_{3}, 90 \mathrm{MHz}\right): \delta(\mathrm{ppm}) 172.0\left(\mathrm{COOCH}_{3}\right), 170.3,170.1$, 169.9, 169.7 (CO), 84.5 (C-1), 74.4, 71.7, 67.1, 67.0 (C-2 to $\mathrm{C}-5)$, 61.4 (C-6), $51.7\left(\mathrm{OCH}_{3}\right), 35.1,25.4\left(\mathrm{CH}_{2}\right), 20.6,20.5(2), 20.4$ $\left(\mathrm{CH}_{3}\right)$; Anal. Calcd for $\mathrm{C}_{18} \mathrm{H}_{26} \mathrm{O}_{11} \mathrm{~S}_{1}$ (450.47): C, 47.99; $\mathrm{H}, 5.82$. Found: ${ }_{\wedge}, 47.85 ; \mathrm{H}, 5.92$.

\subsection{Methyl 3-(2,3,4,6-tetra-O-acetyl- $\beta$-D-} galactopyranosylthio)propanoate $S, S$-dioxide $(21)$

Thiogalactoside 20 ( $1.0 \mathrm{~g}, 2.20 \mathrm{mmol})$ was dissolved in glacial acetic acid $(30 \mathrm{~mL})$. To this soln, Oxone ${ }^{\circledR}(1.90 \mathrm{~g}, 3.30 \mathrm{mmol})$ and NaOAc $(1.20 \mathrm{~g}, 22.0 \mathrm{mmol})$ were added. The mixture was stirred at rt until complete transformation of the starting material (TLC, $1: \hat{1}$ EtOAc-hexane). After filtration it was diluted with water

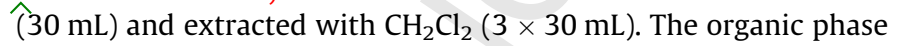
was washed with satd $\mathrm{NaHCO}_{3}(2 \times 30 \mathrm{~mL})$ and water $(1 \times 30 \mathrm{~mL})$, dried, and the solvent was removed under diminished pressure. The crude product was recrystallized from $\mathrm{Et}_{2} \mathrm{O}$ to give $0.74 \mathrm{~g}$ (70\%) of 21 as a white crystalline product. Mp: $120-122{ }^{\circ} \mathrm{C} ;[\alpha]_{\mathrm{D}}$ +6 (c $\left.0.24, \mathrm{CHCl}_{3}\right) ;{ }^{1} \mathrm{H} \mathrm{NMR}\left(\mathrm{CDCl}_{3}, 360 \mathrm{MHz}\right): \delta(\mathrm{ppm}) 5.68(1 \mathrm{H}$, pseudo $\left.\mathrm{t}, J_{2,3} 10.6, J_{1,2} 9.2 \mathrm{~Hz}, \mathrm{H}-2\right), 5.47$ (dd, $1 \mathrm{H}, J_{3,4} 4.0, J_{4,5}$ $2.6 \mathrm{~Hz}, \mathrm{H}-4) 5.16$ (dd, $\left.1 \mathrm{H}, J_{2,3} 10.6, J_{3,4} 4.0 \mathrm{~Hz}, \mathrm{H}-3\right), 4.52(\mathrm{~d}, 1 \mathrm{H}$, $\left.J_{1,2} 9.2 \mathrm{~Hz}, \mathrm{H}-1\right), 4.23-4.16(\mathrm{~m}, 2 \mathrm{H}, \mathrm{H}-5, \mathrm{H}-6), 4.11$ (dd, $1 \mathrm{H}, J_{6,6^{\prime}}$ $\left.11.9, J_{5,6^{\prime}} 5.3 \mathrm{~Hz}, \hat{\mathrm{H}}-6^{\prime}\right), 3.74\left(\mathrm{~s}, 3 \mathrm{H}, \mathrm{OCH}_{3}\right), 3.55(\mathrm{dt}, 1 \mathrm{H}, J$ 7.9, J $\left.\left.6.6 \mathrm{~Hz}, \mathrm{CH}_{2}\right), 3.42 \hat{\mathrm{ddt}}, 1 \mathrm{H}, J 7.9, J 6.6 \mathrm{~Hz}, \mathrm{CH}_{2}\right), 2.92-2.87(\mathrm{~m}, 2 \mathrm{H}$,

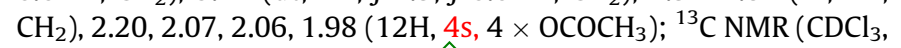
$90 \mathrm{MHz}): \delta(\mathrm{ppm}) 170.5\left(\mathrm{COOCH}_{3}\right), 170.2,169.9,169.8,169.2(\mathrm{CO})$, 88.6 (C-1), 75.4, 71.1, 66.6, 63.3 (C-2 to C-5), 61.0 (C-6), 52.3 $\left(\mathrm{OCH}_{3}\right), 45.1,26.1\left(\mathrm{CH}_{2}\right), 20.6,20.5,20.4,20.3\left(\mathrm{CH}_{3}\right)$; Anal. Calcd for $\mathrm{C}_{18} \mathrm{H}_{26} \mathrm{O}_{13} \mathrm{~S}_{1}$ (482.46): $\mathrm{C}, 44.81 ; \mathrm{H}, 5.43$. Found: $\mathrm{C}, 44.74 ; \mathrm{H}$, 5.46 .
3.16. Mixed salt of $2,3,4,6$-tetra- $O$-acetyl- $\beta$-D-galactopyranosyl1-C-sulfinic acid (22)

Sulfone 21 (0.5 g, $1.05 \mathrm{mmol})$ was dissolved in freshly distilled dry $\mathrm{CHCl}_{3}(15 \mathrm{~mL})$ and DBU $(150 \mu \mathrm{L}, 1.05 \mathrm{mmol})$ was added. The mixture was stirred at rt until complete transformation of the starting material (TLC, $\mathrm{Z}: 3 \mathrm{CHCl}_{3}-\mathrm{MeOH}$ ). The solvent was evaporated and the crude oil was purified by column chromatography $\left(7: 3 \mathrm{CHCl}_{3}-\mathrm{MeOH}\right)$ to give $0.35 \mathrm{~g}$ of 22 as a yellowish oil. ${ }^{1} \mathrm{H}$ NMR $\left(\mathrm{D}_{2} \mathrm{O}, 360 \mathrm{MHz}\right): \delta(\mathrm{ppm}) 5.47\left(\mathrm{dd}, 1 \mathrm{H}, J_{3,4} 4.0, J_{4,5} 1.0 \mathrm{~Hz}\right.$, $\mathrm{H}-4), 5.43\left(\mathrm{t}, 1 \mathrm{H}, J_{1,2} 10.6, J_{2,3} 10.6 \mathrm{~Hz}, \mathrm{H}-2\right), 5.26$ (dd, $1 \mathrm{H}, J_{2,3}$ 10.6, J J,4 $4.0 \mathrm{~Hz}, \mathrm{H}-3$ ), 3.64 (d, $1 \mathrm{H}, J_{1,2} 10.6 \mathrm{~Hz}, \mathrm{H}-1$ ), 3.55-3.45 (m, 2H, H-6, 其-6'), 3.29 (pseudo t, $1 \mathrm{H}, J_{5,6} 6.6, J_{56^{\prime}} 6.6 \mathrm{~Hz}, \mathrm{H}-5$ ), $2.21,2.06(2), 2.00\left(12 \mathrm{H}, 3 \mathrm{~s}, 4 \times \mathrm{OCOCH}_{3}\right) ;{ }^{13} \mathrm{C}$ NMR $\left(\mathrm{D}_{2} \mathrm{O}\right.$, $90 \mathrm{MHz}): \delta(\mathrm{ppm}) 173.6,173.4,173.0,172.9$ (CO), 92.8 (C-1), 74.4, 72.4, 68.3, 65.4 (C-2 to C-5), 62.1 (C-6), 20.4, 20.2, 20.1, $20.0\left(\mathrm{CH}_{3}\right)$. For further preparative purposes, the crude product obtained after solvent removal was used without purification.

\subsection{7. $2,3,4,6$-Tetra- 0 -acetyl- $\beta$-D-galactopyranosyl-1-C- sulfonamide (23)}

Sulfinate salt $22(\sim 0.35 \mathrm{~g})$ was suspended in water $(5 \mathrm{~mL})$. $\mathrm{H}_{2} \mathrm{NOSO}_{3} \mathrm{H}(0.28 \mathrm{~g}, 2.5 \mathrm{mmol})$ and $\mathrm{NaOAc}(0.24 \mathrm{~g}, 2.92 \mathrm{mmol})$ were added and the mixture was stirred at $\mathrm{rt}$ for $2 \mathrm{~h}$. After extraction with EtOAc $(5 \times 10 \mathrm{~mL})$, the organic phase was washed with satd $\mathrm{NaHCO}_{3}(2 \times 10 \mathrm{~mL})$ and water $(10 \mathrm{~mL})$, dried, and the solvent e्रvaporated to give $0.27 \mathrm{~g}$ ( $79 \%$, from the sulfone, $0.4 \mathrm{~g}$ ) of $\mathbf{2 3}$ as a yellowish oil. $R_{\mathrm{f}}=0.23$ (1:1 EtOAc-hexane); $[\alpha]_{\mathrm{D}}+32$ (c $0.22, \mathrm{Q} 1$ $\left.\mathrm{CHCl}_{3}\right) ;{ }^{1} \mathrm{H} \mathrm{NMR}\left(\mathrm{CDCl}_{3}, 360 \mathrm{MHz}\right): \delta(\mathrm{ppm}) 5.51\left(\mathrm{~s}, 1 \mathrm{H}, \mathrm{NH}_{2}\right)$, $5.48\left(1 \mathrm{H}, \mathrm{dd}, J_{3,4} 4.0, J_{4,5} 1.0 \mathrm{~Hz}, \mathrm{H}-4\right), 5.29\left(\mathrm{~s}, 1 \mathrm{H}, \mathrm{NH}_{2}\right), 5.26(\mathrm{t}$, $1 \mathrm{H}, J_{1,2} 10.6, J_{2,3} 10.6 \mathrm{~Hz}, \mathrm{H}-2$ ), 5.17 (dd, $1 \mathrm{H}, J_{2,3} 10.6, J_{3,4} 2.3 \mathrm{~Hz}$, $\mathrm{H}-3), 4.41$ (d, 1H, $\left.J_{1,2} 10.6 \mathrm{~Hz}, \mathrm{H}-1\right), 4.22$ (t, $1 \mathrm{H}, J_{5,6} 6.6, J_{5,6^{\prime}}$ $6.6 \mathrm{~Hz}, \mathrm{H}-5), 4.16-4.11$ (m, 2H, H-6, H-6'), 3.93-3.86 (m, 3H, H-5, $\left.\mathrm{NH}_{2}\right), 2.17,2.08,2.05,1.99\left(12 \mathrm{H}, 4 \mathrm{~s}, 4 \times \mathrm{OCOCH}_{3}\right) ;{ }^{13} \mathrm{C} \mathrm{NMR}\left(\mathrm{CDCl}_{3}\right.$, $90 \mathrm{MHz}): \delta(\mathrm{ppm}) 171.1,170.5,170.0,169.8(\mathrm{CO}), 87.8(\mathrm{C}-1), 75.2$, 70.7, 66.9, 65.3 (C-2 to C-5), 60.9 (C-6), 20.8, $20.6(2), 20.5\left(\mathrm{CH}_{3}\right)$. Anal. Calcd for $\mathrm{C}_{14} \mathrm{H}_{21} \mathrm{O}_{11} \mathrm{NS}$ (411.39): $\mathrm{C}^{\mathrm{N}}$, 40.88; H, 5.15. Found: $\mathrm{C}^{\text {, }}$ 40.80; H, 5.21.

\subsection{Bromination methods}

(a) A methyl 3-(2,3,4,6-tetra-O-acetyl- $\beta$-D-glycopyranosylthio)propanoate $S, S$-dioxide (11 or $\mathbf{2 1}, \hat{0} .1 \mathrm{~g}, 0.21 \mathrm{mmol}$ ) was dissolved in dry $\mathrm{CCl}_{4}(10 \mathrm{~mL})$, and bromine $(30 \mu \mathrm{L}, 0.73 \mathrm{mmol})$ and some $\mathrm{K}_{2} \mathrm{CO}_{3}$ were added. The mixture was placed in an Erlenmeyer flask above a heat lamp (375 W, distance from the lamp $\sim 2-3 \mathrm{~cm}$, height of the soln 1-2 cm), and refluxed. It was monitored by (TLC, $1: 1$ EtOAc-hexane). After $3 \mathrm{~h} \mathrm{CHCl}_{3}(10 \mathrm{~mL})$ was added, and the mixture washed with $1 \mathrm{M}$ aq $\mathrm{Na}_{2} \mathrm{~S}_{2} \mathrm{O}_{3}(10 \mathrm{~mL})$, satd $\mathrm{NaHCO}_{3}$ $(2 \times 10 \mathrm{~mL})$ and water $(10 \mathrm{~mL})$. After drying, the solvent was removed and the residue purified by column chromatography $(1: 1$ EtOAc-hexane).

(b) A 2,3,4,6-tetra- $O$-acetyl- $\beta$-D-glycopyranosyl-1- $C$-sulfonamide (14 or $23,0.1 \mathrm{~g}, 0 . \hat{24} \mathrm{mmol}$ ) was dissolved in dry $\mathrm{CHCl}_{3}$ $(10 \mathrm{~mL})$, and bromine $(30 \mu \mathrm{L}, 0.73 \mathrm{mmol})$ and some $\mathrm{K}_{2} \mathrm{CO}_{3}$ were added. The mixture was placed in an Erlenmeyer flask above a heat lamp (375 W, distance from the lamp $\sim 2-3 \mathrm{~cm}$, height of the soln $1-2 \mathrm{~cm})$, and refluxed. If the mixture decolourized, bromine $(10 \mu \mathrm{L})$ was added again. It was monitored by (TLC, $1: 1$ EtOAc-hexane). After $8 \mathrm{~h} \mathrm{CHCl}_{3}(10 \mathrm{~mL})$ was added, and the mixture washed with $1 \mathrm{M}$ aq $\mathrm{Na}_{2} \mathrm{~S}_{2} \mathrm{O}_{3}(10 \mathrm{~mL})$, satd $\mathrm{NaHCO}_{3}(2 \times 10 \mathrm{~mL})$ and water $(10 \mathrm{~mL})$. After drying, the solvent was removed and the residue purified by column chromatography (1:个EtOAc-hexane).

(c) 2,3,4,6-Tetra- $O$-acetyl- $\beta$-D-glycopyranosyl-1-C-sulfonamide $\left(14,0.1 \mathrm{~g}, 0.24 \mathrm{mmol}\right.$ ) was dissolved in dry $\mathrm{PhCF}_{3}(10 \mathrm{~mL})$, and 
bromine ( $30 \mu \mathrm{L}, 0.73 \mathrm{mmol}$ ) and some $\mathrm{K}_{2} \mathrm{CO}_{3}$ were added. The mixture was placed in an Erlenmeyer flask above a heat lamp ( $375 \mathrm{~W}$, distance from the lamp $\sim 2-3 \mathrm{~cm}$, height of the soln $1-2 \mathrm{~cm}$ ), and refluxed. It was monitored by (TLC, $\lambda: 1$ EtOAc-hexane). After 4 h, the mixture was washed with $1 \mathrm{M}$ 주 $\mathrm{Na}_{2} \mathrm{~S}_{2} \mathrm{O}_{3}(10 \mathrm{~mL})$, satd $\mathrm{NaH}-$ $\mathrm{CO}_{3}(2 \times 10 \mathrm{~mL})$ and water $(10 \mathrm{~mL})$. After drying, the solvent was removed under diminished pressure, and the residue purified by column chromatography $\mathrm{\lambda}_{1}: 1$ EtOAc-hexane).

\subsection{Methyl $\left(2,3,4,6\right.$-tetra- $O$-acetyl-1- $C_{- \text {-bromo- } \beta-D-}$ glucopyranosylthio)propanoate $S, S$-dioxide (24)}

Isolated from the bromination mixture of $11(1.0 \mathrm{~g}, 2.07 \mathrm{mmol})$ obtained by method (a) as a white crystalline product $(0.25 \mathrm{~g}, 21 \%)$ from hexane. Mp: $22-93{ }^{\circ} \mathrm{C} ;[\alpha]_{\mathrm{D}}+176\left(\mathrm{c} 0.28, \mathrm{CHCl}_{3}\right) ;{ }^{1} \mathrm{H}$ NMR $\left(\mathrm{CDCl}_{3}, 360 \mathrm{MHz}\right.$.): $\delta$ (ppm) 5.66 (d, $\left.1 \hat{\mathrm{H}}, J_{1,2} 9.2 \mathrm{~Hz}, \mathrm{H}-2\right), 5.49$, 5.25 (pseudo t, $2 \mathrm{H}, J \sim 9.2 \mathrm{~Hz}$ in each, $\mathrm{H}-3, \mathrm{H}-4), 4.36-4.18$ (m, $\left.3 \mathrm{H}, \mathrm{H}-5, \mathrm{H}-6, \mathrm{H}-6^{\prime}\right), 3.75\left(\mathrm{~s}, 3 \mathrm{H}, \mathrm{OCH}_{3}\right), 3.72-3.63\left(\mathrm{~m}, 2 \mathrm{H}, \mathrm{CH}_{2}\right)$, 2.89-2.84 (m, 2H, $\left.\mathrm{CH}_{2}\right), 2.12,2.09,2.06,2.02(12 \mathrm{H}, 4 \mathrm{~s}$,

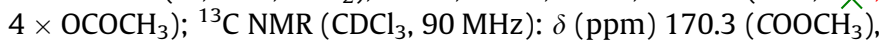
170.2, 169.6, 168.9, 168.6 (CO), 106.8 (C-1), 75.4, 71.3, 66.9, 66.2 (C-2 to C-5), 60.2 (C-6), $52.4\left(\mathrm{OCH}_{3}\right), 43.1,26.3\left(\mathrm{CH}_{2}\right), 20.4(3)$ $20.3\left(\mathrm{CH}_{3}\right)$. Anal. Calcd for $\mathrm{C}_{18} \mathrm{H}_{25} \mathrm{O}_{13} \mathrm{~S}_{1}$ (561.36): C. 38.50; $\mathrm{H}$, 4.49; S, 5.71. Found: C. 38.55; H, 4.42; S, 5.77.

\section{3.20. Methyl (2,3,4,6-tetra-O-acetyl-5-C-bromo- $\beta$-D-} glucopyranosylthio)propanoate $S, S$-dioxide (25)

Isolated from the bromination mixture of $\mathbf{1 1}(1.0 \mathrm{~g}, 2.07 \mathrm{mmol})$ by method (a) as a colourless oil $(0.35 \mathrm{~g}, 30 \%) . R_{\mathrm{f}}=0.66$ (1:1 EtOAchexane); $[\alpha]_{\mathrm{D}}-252$ (c $\left.0.20, \mathrm{CHCl}_{3}\right) ;{ }^{1} \mathrm{H} \mathrm{NMR}\left(\mathrm{CDCl}_{3}, 360 \mathrm{MHz}\right): \delta$ (ppm) 5.66, 5.58 (pseudo t, 2H, $J \sim 9.2 \mathrm{~Hz}$ in each, H-2, H-3), 5.26 (d, $1 \mathrm{H}, J_{1,2} 10.6 \mathrm{~Hz} . \mathrm{H}-1$ ), 4.96 (d, 1H, $\left.J_{3,4} 9.2 \mathrm{~Hz}, \mathrm{H}-4\right), 4.58$ (d, $1 \mathrm{H}$, $\left.J_{6,6^{\prime}} 11.9 \mathrm{~Hz}, \mathrm{H}-6\right), 4.46\left(\mathrm{~d}, 1 \mathrm{H}, J_{\mathrm{K}, 6^{\prime}} 11.9 \mathrm{~Hz}, \mathrm{H}_{-} 6^{\prime}\right), 3.76(\mathrm{~s}, 3 \mathrm{H}$, $\left.\mathrm{OCH}_{3}\right), 3.82-3.54\left(\mathrm{~m}, 2 \mathrm{H}, \mathrm{CH}_{2}\right), 2.88-2.84\left(\mathrm{~m}, 2 \mathrm{H}, \mathrm{CH}_{2}\right), 2.13$, $2.10,2.07,2.03\left(12 \mathrm{H}, 4 \mathrm{~s}, 4 \times \mathrm{OCOCH}_{3}\right) ;{ }^{13} \mathrm{C} \quad \mathrm{NMR}\left(\mathrm{CDCl}_{3}\right.$, $90 \mathrm{MHz}): \delta(\mathrm{ppm}) 170.1\left(\mathrm{COOCH}_{3}\right), 169.6,169.5,168.9,168.7$ (CO), 98.3 (C-5), 85.5 (C-1), 71.2, 67.9, 66.9, (C-2 to C-4), 60.2 (C6), $52.4\left(\mathrm{OCH}_{3}\right), 45.9,26.1\left(\mathrm{CH}_{2}\right), 20.4(2) 20.3(2)\left(\mathrm{CH}_{3}\right)$. Anal. Calcd for $\mathrm{C}_{18} \mathrm{H}_{25} \mathrm{O}_{13} \mathrm{~S}_{1}$ (561.36): ${ }_{\wedge}^{C}, 38.50 ; \mathrm{H}, 4.49$. Found: ${ }_{\Lambda}^{\mathrm{C}}, 38.48 ; \mathrm{H}$, 4.45.

\subsection{Methyl (2,3,4,6-tetra-O-acetyl-1-C-bromo- $\beta$-D-} galactopyranosylthio)propanoate $S$,S-dioxide (26)

Isolated from the bromination mixture of 21 ( $0.20 \mathrm{~g}$, $0.41 \mathrm{mmol}$ ) obtained by method (a) as a colourless oil $(0.1 \mathrm{~g}$, $43 \%) . R_{\mathrm{f}}=0.55$ (1:1 EtOAc-hexane); $[\alpha]_{\mathrm{D}}+154\left(\right.$ c $\left.0.18, \mathrm{CHCl}_{3}\right) ;{ }^{1} \mathrm{H}$ NMR $\left(\mathrm{CDCl}_{3}, 360 \mathrm{MHz}\right): \delta(\mathrm{ppm}) 5.82\left(\mathrm{~d}, 1 \mathrm{H}, \mathrm{J}_{1,2} 10.6 \mathrm{~Hz}, \mathrm{H}-2\right)$, $5.55\left(\mathrm{dd}, 1 \mathrm{H}, J_{3,4} 2.6, J_{4,5} 1.0 \mathrm{~Hz}, \mathrm{H}-4\right), 5.32$ (dd, $1 \mathrm{H}, J_{2,3} 10.6, J_{3,4}$ $2.6 \mathrm{~Hz}, \mathrm{H}-3), 4.52$ (t, $\left.1 \mathrm{H}, J_{5,6} 6.6, J_{5,6} 6.6 \mathrm{~Hz}, \mathrm{H}-5\right), 4.26-4.22$ (m, $\left.2 \mathrm{H}, \mathrm{H}-6, \mathrm{H}-6^{\prime}\right), 3.75\left(\mathrm{~s}, 3 \mathrm{H}, \mathrm{OCH}_{3}\right), 3.72-3.66\left(\mathrm{~m}, 2 \mathrm{H}, \mathrm{CH}_{2}\right), 2.96-$ $2.92\left(\mathrm{~m}, \hat{2} \mathrm{H}, \mathrm{CH}_{2}\right), 2.12,2.07,2.05,2.00\left(12 \mathrm{H}, 4 \mathrm{~s}, 4 \times \mathrm{OCOCH}_{3}\right)$; ${ }^{13} \mathrm{C} \mathrm{NMR}\left(\mathrm{CDCl}_{3}\right) \delta(\mathrm{ppm}): 170.2\left(\mathrm{COOCH}_{3}\right), 172.0,170.1,169.7$, 169.6 (CO), 108.1 (C-1), 74.8, 69.6, 66.1, 63.8 (C-2 to C-5), 60.3 (C-6), $52.4\left(\mathrm{OCH}_{3}\right), 43.0,26.3\left(\mathrm{CH}_{2}\right), 20.920 .4(3)\left(\mathrm{CH}_{3}\right)$. Anal. Calcd for $\mathrm{C}_{18} \mathrm{H}_{25} \mathrm{O}_{13} \mathrm{~S}_{1}$ (561.36): C, 38.50; H, 4.49; S, 5.71. Found: C, 38.53; $\mathrm{H}, 4.41 ; \mathrm{S}, 5.77$.

\subsection{Methyl (2,3,4,6-tetra-0-acetyl-5-C-bromo- $\beta$-D-} galactopyranosylthio)propanoate $S_{\wedge}, S$-dîxide (27)

Isolated from the bromination mixture of $21(0.20 \mathrm{~g}$, $0.41 \mathrm{mmol})$ obtained by method (a) as a colourless oil $(0.06 \mathrm{~g}$, $27 \%) . R_{\mathrm{f}}=0.50$ (1:1 EtOAc-hexane); $[\alpha]_{\mathrm{D}}-131\left(\right.$ c $\left.0.18, \mathrm{CHCl}_{3}\right) ;{ }^{1} \mathrm{H}$ NMR $\left(\mathrm{CDCl}_{3}, 360 \mathrm{MHz}\right): \delta(\mathrm{ppm}) 5.80-\hat{5} .70(\mathrm{~m}, 2 \mathrm{H}, \mathrm{H}-2, \mathrm{H}-3)$,
5.63 (dd, $\left.1 \mathrm{H}, J_{3,4} 2.6, J_{4,5} 1.0 \mathrm{~Hz}, \mathrm{H}-4\right), 4.92$ (d, $1 \mathrm{H}, J_{1,2} 9.2 \mathrm{~Hz} . \mathrm{H}-$ 1), $4.67\left(\mathrm{~d}, 1 \mathrm{H}, J_{\text {风6 }} 11.9 \mathrm{~Hz}, \mathrm{H}-6\right), 4.40\left(\mathrm{~d}, 1 \mathrm{H}, J_{\text {ब }} 6^{\prime} 11.9 \mathrm{~Hz}, \mathrm{H}^{\prime}-6^{\prime}\right)$, $3.73\left(3 \mathrm{H}, \mathrm{s}, \mathrm{OCH}_{3}\right), 3.46-3.40\left(2 \mathrm{H}, \mathrm{m}, \mathrm{CH}_{2}\right), 2.89-2.82(2 \mathrm{H}, \mathrm{m}$,

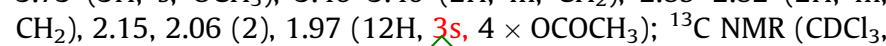
$90 \mathrm{MHz}): \delta(\mathrm{ppm}) 170.2\left(\mathrm{COOCH}_{3}\right), 169.5(2), 169.0$ (2) (CO), 95.7 (C-5), 85.9 (C-1), 69.8, 68.5, 65.9, (C-2 to C-4), 60.3 (C-6), 52.5 $\left(\mathrm{OCH}_{3}\right), 45.8,26.0\left(\mathrm{CH}_{2}\right), 20.9,20.5,20.4,20.3\left(\mathrm{CH}_{3}\right)$. Anal. Calcd for $\mathrm{C}_{18} \mathrm{H}_{25} \mathrm{O}_{13} \mathrm{~S}_{1}$ (561.36): C. 38.50; H, 4.49. Found: C. 38.59; $\mathrm{H}$, 4.53 .

\subsection{3. $2,3,4,6$-Tetra-O-acetyl-1-C-bromo- $\beta$-D-glucopyranosyl-1-C- sulfonamide (28)}

Obtained by bromination of $\mathbf{1 4}(0.60 \mathrm{~g}, 1.45 \mathrm{mmol})$ by method (b) $0.05 \mathrm{~g}(11 \%)$ and method (c) $0.058 \mathrm{~g}(12 \%)$ as a white crystalline product from hexane. Mp: $185-187^{\circ} \mathrm{C} ;[\alpha]_{\mathrm{D}}+235$ ( $\mathrm{c} 0.26, \mathrm{CHCl}_{3}$ ); ${ }^{1} \mathrm{H}$ NMR $\left(\mathrm{CDCl}_{3}, 360 \mathrm{MHz}\right): \delta(\mathrm{ppm}) 5.56-5.45(\mathrm{~m}, 2 \mathrm{H}, \mathrm{H}-2, \mathrm{H}-3$ or $\mathrm{H}-4), 5.38-5.34\left(\mathrm{~m}, 2 \mathrm{H}, \mathrm{NH}_{2}\right), 5.26(\mathrm{t}, 1 \mathrm{H}, J 9.2, J 9.2 \mathrm{~Hz}, \mathrm{H}-4$ or $\mathrm{H}-3), 4.33-4.26$ (m, 3H, H-5, H-6, H-6'), 2.10 (2), 2.05, $2.01(12 \mathrm{H}$, $\left.4 \mathrm{~s}, 4 \times \mathrm{OCOCH}_{3}\right) ;{ }^{13} \mathrm{C} \mathrm{NMR}\left(\mathrm{CDCl}_{3}, 90 \mathrm{MHz}\right): \delta(\mathrm{ppm}) 170.9$, 169.8, 169.7, 169.3 (CO), 105.4 (C-1), 75.2, 71.5, 68.7, 66.6 (C-2 to C-5), 60.4 (C-6), 20.7 (2), 20.5, $20.4\left(\mathrm{CH}_{3}\right)$. Anal. Calcd for $\mathrm{C}_{14} \mathrm{H}_{20} \mathrm{O}_{11} \mathrm{BrNS}$ (490.28): . . 34.30; H, 4.11. Found: C. $34.35 ; \mathrm{H}, 4.01$.

\subsection{2,3,4,6-Tetra-O-acetyl-5-C-bromo- $\beta$-D-glucopyranosyl-1-C- sulfonamide (29)}

Obtained by bromination of $\mathbf{1 4}(0.60 \mathrm{~g}, 1.45 \mathrm{mmol})$ by method (b) $0.054 \mathrm{~g}(12 \%)$ and method (c) $0.056 \mathrm{~g} \mathrm{(12 \% )} \mathrm{as} \mathrm{a} \mathrm{yellowish} \mathrm{oil.}$ $R_{\mathrm{f}}=0.59$ (1:1 EtOAc-hexane); $[\alpha]_{\mathrm{D}}-284\left(\mathrm{c} 0.24, \mathrm{CHCl}_{3}\right) ;{ }^{1} \mathrm{H} \mathrm{NMR}$ $\left(\mathrm{CDCl}_{3}, 360 \mathrm{MHz}\right): \delta(\mathrm{ppm}) 5.58,5.51$ (pseudo t, $2 \mathrm{H}, J \sim 9.2 \mathrm{~Hz}$ in each, $\mathrm{H}-2, \mathrm{H}-3), 5.38-5.32\left(\mathrm{~m}, 2 \mathrm{H}, \mathrm{NH}_{2}\right), 5.23\left(\mathrm{~d}, 1 \mathrm{H}, J_{1,2} 9.2, \mathrm{~Hz}\right.$, $\mathrm{H}-1$ ), 4.84 (d, 1H, J3,4 $9.2 \mathrm{~Hz}, \mathrm{H}-4), 4.90-4.56$ (m, 2H, H-6, H-6'), 2.11, 2.08 (2), $2.01\left(12 \mathrm{H}, 3 \mathrm{~s}, 4 \times \mathrm{OCOCH}_{3}\right) ;{ }^{13} \mathrm{C} \mathrm{NMR}\left(\mathrm{CDCl}_{3}\right.$, $90 \mathrm{MHz}): \delta(\mathrm{ppm}) 170.2(2), 169.6,169.0(\mathrm{CO}), 98.4$ (C-5), 85.6 (C-1), 71.1, 68.2, 67.0 (C-2 to C-4), 65.5 (C-6), 20.6 (2), 20.4 (2) $\left(\mathrm{CH}_{3}\right)$. Anal. Calcd for $\mathrm{C}_{14} \mathrm{H}_{20} \mathrm{O}_{11}$ BrNS (490.28): C, 34.30; H, 4.11. Found: C, 34.28; H, 4.08.

\section{Acknowledgements}

Financial support for this work was provided by the Hungarian Scientific Research Fund (Grants: OTKA 46081 and 61336). The authors thank P. Gergely and T. Docsa for the glycogen phosphorylase assays.

\section{References}

1. Somsák, L.; Nagy, V.; Docsa, T.; Tóth, B.; Gergely, P. Tetrahedron: Asymmetry 2000, 11, 405-408.

2. Somsák, L.; Nagy, V. Tetrahedron: Asymmetry 2000, 11, 1719-1727. Corrigendum 2247

3. Somsák, L.; Kovács, L.; Tóth, M.; Ősz, E.; Szilágyi, L.; Györgydeák, Z.; Dinya, Z.; Docsa, T.; Tóth, B.; Gergely, P. J. Med. Chem. 2001, 44, 2843-2848.

4. Czifrák, K.; Kovács, L.; Kövér, K. E.; Somsák, L. Carbohydr. Res. 2005, 340, 2328 2334.

5. Somsák, L.; Nagy, V.; Hadady, Z.; Docsa, T.; Gergely, P. Curr. Pharm. Des. 2003, 9, 1177-1189.

6. Somsák, L.; Nagy, V.; Hadady, Z.; Felföldi, N.; Docsa, T.; Gergely, P. In Frontiers in Medicinal Chemistry; Reitz, A. B., Kordik, C. P., Choudhary, M. I., Rahman, A. u., Eds.; Bentham, 2005; pp 253-272.

7. Oikonomakos, N. G.; Somsák, L. Curr. Opin. Invest. Drugs 2008, 9, 379-395.

8. Somsák, L.; Czifrák, K.; Tóth, M.; Bokor, É.; Chrysina, E. D.; Alexacou, K. M.; Q2 $2_{710}$ Hayes, J. M.; Tiraidis, C.; Lazoura, E.; Leonidas, D. D.; Zographos, S. E.; Oikonomakos, N. G. Curr. Med. Chem., in press.

9. Hopfinger, A. J.; Reaka, A.; Venkatarangan, P.; Duca, J. S.; Wang, S. J. Chem. Inf.

10. Knapp, S.; Darout, E.; Amorelli, B. J. Org. Chem. 2006, 71, 1380-1389.

11. Lipták, A. In Progress in Biological Chirality; Pályi, G., Zucchi, C., Cagliotti, L., Eds.; Elsevier, 2004; pp 181-202.

12. Lipták, A.; Borbás, A. Magy. Kém. Foly. 2004, 109-110, 60-63. Comp. Sci. 1999, 39, 1151-1160. 
13. Lipták, A.; Balla, E.; Jánossy, L.; Sajtos, F.; Szilágyi, L. Tetrahedron Lett. 2004, 45, 839-842.

14. Lipták, A.; Csávás, M.; Borbás, A. 2008, unpublished results.

15. Bonner, W. A. J. Am. Chem. Soc. 1951, 73, 2659-2666.

16. Sanhueza, C. A.; Dorta, R. L.; Vazquez, J. T. Tetrahedron: Asymmetry 2008, 19 258-264.

17. Černý, M.; Vrkoč, J.; Staněk, J. Collect. Czech. Chem. Commun. 1959, 24 64-69.

18. Horton, D. Meth. Carbohydr. Chem. 1963, 2, 433-437.

19. Graham, S. L.; Scholz, T. H. Synthesis 1986, 1031-1032.

20. Baskin, J. M.; Wang, Z. Tetrahedron Lett. 2002, 43, 8479-8483.

730 21. Chan, W. Y.; Berthelette, C. Tetrahedron Lett. 2002, 43, 4537-4540.

22. Sokolov, S. D.; Savochkina, L. P.; Kochetkov, N. K. Zh. Obsh. Khim. 1964, 34, 4099-4103.

23. Sridhar, P. R.; Prabhu, K. R.; Chandrasekaran, S. Eur. J. Org. Chem. 2004, 48094815.

24. Micskei, K.; Juhász, Z.; Ratkovic, Z.; Somsák, L. Tetrahedron Lett. 2006, 47, 61176120.

25. Černý, M.; Staněk, J.; Pacák, J. Monatsh. Chem. 1963, 94, 290-294.
26. Frgala, J.; Černý, M.; Staněk, J. Collect. Czech. Chem. Commun. 1975, 40, 14111425.

27. Somsák, L.; Ferrier, R. J. Adv. Carbohydr. Chem. Biochem. 1991, 49, 37-92.

28. Praly, J. P.; Descotes, G. Tetrahedron Lett. 1990, 31, 1133-1136.

29. Praly, J.-P.; Somsák, L.; Mahmoud, S. H.; El Kharraf, Z.; Descotes, G.; Farkas, I. J. Carbohydr. Chem. 1992, 11, 201-216.

30. Czifrák, K.; Somsák, L. Tetrahedron Lett. 2002, 43, 8849-8852.

31. Merényi, R.; Janousek, Z.; Viehe, H. G. In Substituent Effects in Radical Chemistry; Viehe, H. G., Janousek, Z., Merényi, R., Eds.; Reidel, D.: Dordrecht, 1986; pp 301324.

32. Kiss, L.; Somsák, L. Carbohydr. Res. 1996, 291, 43-52.

33. Czifrák, K.; Szilágyi, P.; Somsák, L. Tetrahedron: Asymmetry 2005, 16, 127-141.

34. Praly, J.-P. Adv. Carbohydr. Chem. Biochem. 2001, 56, 65-151.

35. Ősz, E.; Sós, E.; Somsák, L.; Szilágyi, L.; Dinya, Z. Tetrahedron 1997, 53, 58135824

36. Ösz, E.; Szilágyi, L.; Somsák, L.; Bényei, A. Tetrahedron 1999, 55, 2419-2430.

37. Watson, K. A.; Mitchell, E. P.; Johnson, L. N.; Son, J. C.; Bichard, C. J. F.; Orchard, M. G.; Fleet, G. W. J.; Oikonomakos, N. G.; Leonidas, D. D.; Kontou, M.; Papageorgiou, A. Biochemistry 1994, 33, 5745-5758. 